\title{
Musei tra narrazione, visualità e new media
}

\author{
Tommaso Empler \\ Adriana Caldarone \\ Alexandra Fusinetti
}

\section{Abstract}

II panorama museale, dal punto di vista della comunicazione dei contenuti, presenta due estremi: il museo 'tradizionale', spazio reale in cui il visitatore acquisisce passivamente informazioni selezionate attraverso un linguaggio scelto a priori, e il museo 'multimediale', spazio virtuale studiato per rendere attivo e partecipato il ruolo del visitatore che sceglie il proprio percorso di visita e seleziona autonomamente le informazioni che vuole acquisire.

Tra i due vi è una particolare tipologia di museo che è possibile definire 'transmediale' in cui sono presenti diversi strumenti e linguaggi di comunicazione che trasformano i processi alla base dell'apprendimento e i livelli di attenzione. Fanno tutti riferimento al campo dell'edutainment e possono essere riassunti in: interactive experience, immersive experience, serious games, gamification e universal design. In questi musei emittente e ricevente sono collegati mediante reciproche interrelazioni e correlazioni.

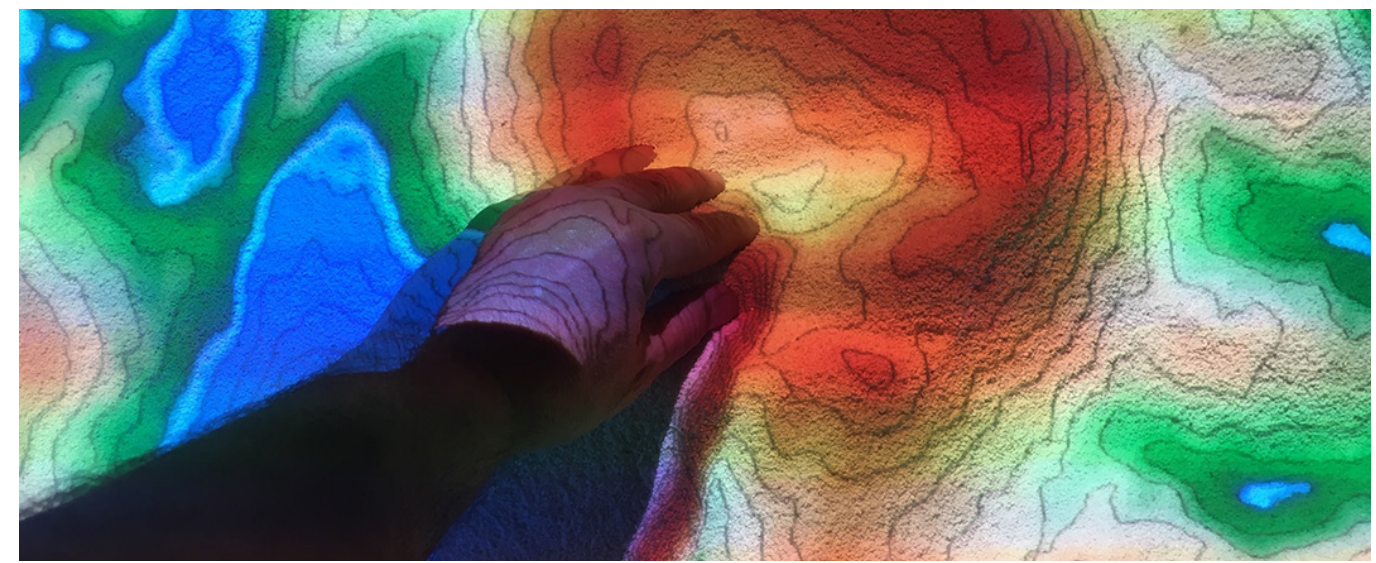




\section{Introduzione}

La museografia passa dalla tradizionale organizzazione dei musei settecenteschi, quando nasce la disciplina, ai recenti musei virtuali.

I musei tradizionali erano inizialmente strutturati secondo la logica delle Wunderkammer, camere delle meraviglie in cui erano raccolti oggetti straordinari. Al giorno d'oggi il concetto di 'museo', sia secondo quanto esposto nelle Linee Guida per la comunicazione nei musei [Da Milano, Sciacchitano 2015], sia secondo la nuova definizione proposta dall'ICOM, è associato alla diffusione della conoscenza e al ruolo educativo che ne scaturisce. I depositari delle informazioni, in ambito museale, sono le figure del direttore o del curatore, che spesso si confrontano con la figura del visual designer per scegliere la corretta forma di rappresentazione che veicoli correttamente il messaggio e la conoscenza. Poiché nell'esposizione l'oggetto è estrapolato dal proprio contesto d'origine, utile alla sua corretta interpretazione semantica, è necessario che gli apparati comunicativi siano di supporto per incrementarne la

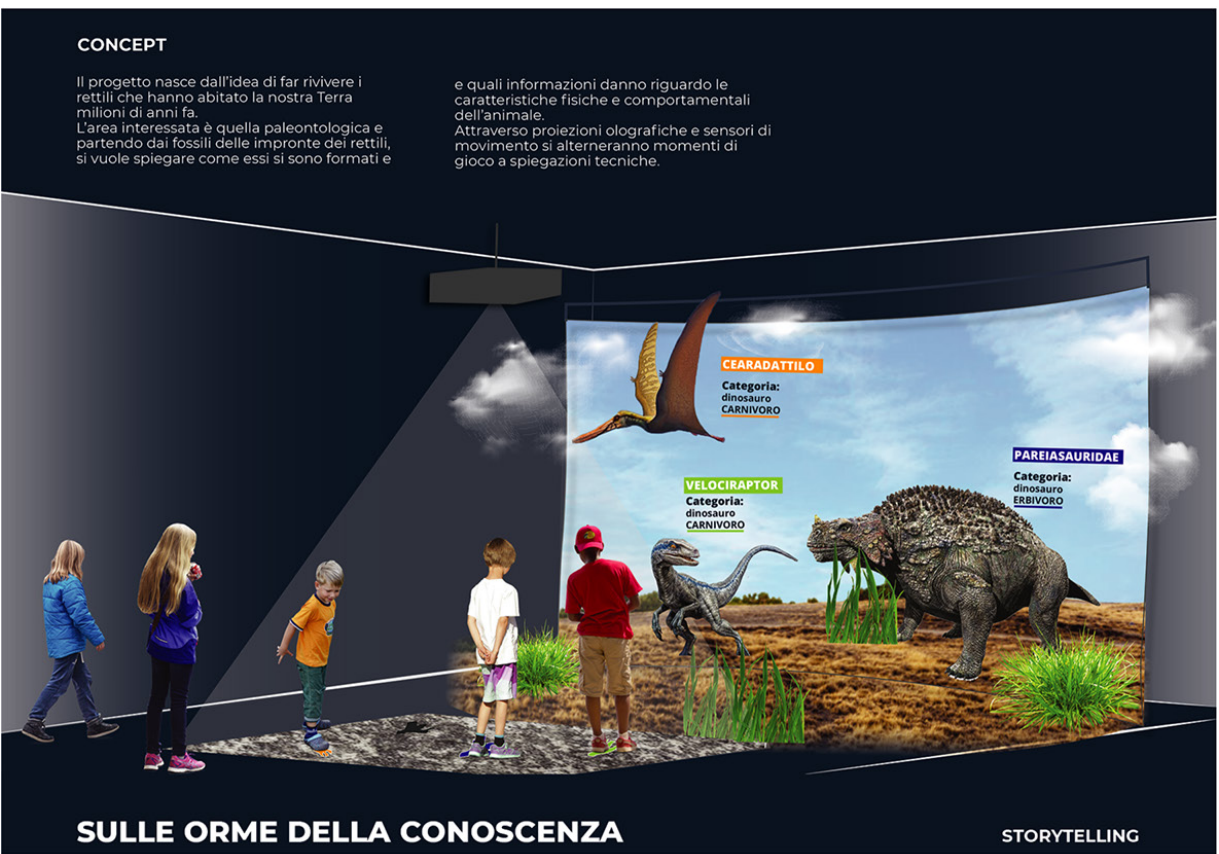

SULLE ORME DELLA CONOSCENZA

STORYTELLING
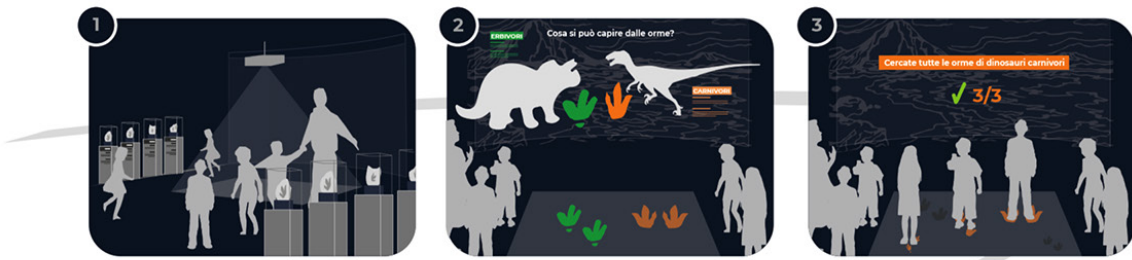

Massimo 10 persone entrano nella proiezione a terra. Interno a loro sono le teche con gli icnofossili.

Spiegazione di cosa si può capire degli animali attraverso le loro orme fossili (es. erbivori o carnivori, bipedi

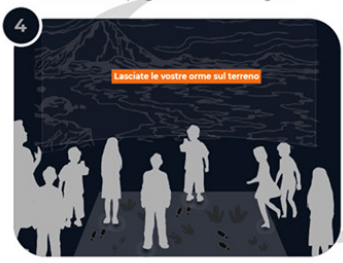

Un gruppo di dinosauri è passato sul
terreno! Anche i bambini sono invitati terreno! Anchei bambini sonoin starare capire tutto il processo di trasformazione che va dall' impront

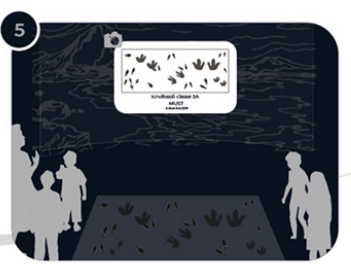

Come ricordo dell' esperienza, il pavimento conle orme dei bambini consegnara alla fine del percorso museale.
Una volta finita la spiegazione vengo
no proposti ai bambini una serie di giochiper riconosscere le varie orme, scere le orme di carnivori) max4 4 .

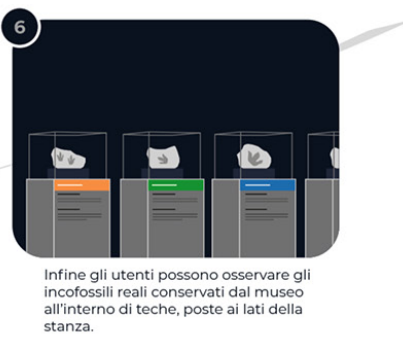


Fig. 2. Il serious game, Meteorite Hunters si pone l'obiettivo di informare ed educare adulti e bambini riguardo il mondo delle meteoriti. Nelle vesti di geologi i giocatori potranno scoprire tutte le curiosità e le proprietà delle meteoriti precipitate sulla terra andando alla ricerca di veri ancropri campioni in un e propricampioni in un a pica sara pietra sara custodita in un backpack, e, piu meteoriti saranno trovati, maggiore sara il punteggio. II tutto sarà accompagnato da un quiz a cui i giocatori dovranno rispondere per proseguire il gioco e ottenere le meteoriti (studenti: Pignatone, Laurini, Pilato). comprensione [Antinucci 20 14]. Un metodo efficace di cui si serve oggi la comunicazione in ambito museale è lo storytelling, tecnica di comunicazione che, attraverso il racconto, cerca il coinvolgimento e l'attenzione del pubblico per veicolare il messaggio.

L'evoluzione tecnologica ha definito il transmedia storytelling [Jenkins 20 I4]: una stessa storia raccontata su diversi media e piattaforme, nella quale ogni singolo strumento o linguaggio comunicativo offre un contributo specifico all'intero complesso narrativo. Non si tratta di ripetere il racconto principale su un altro media, ma di affidare parte della narrazione a vari media, coinvolgendo così anche diverse tipologie di spettatori [Treccani 2020, ad vocem].

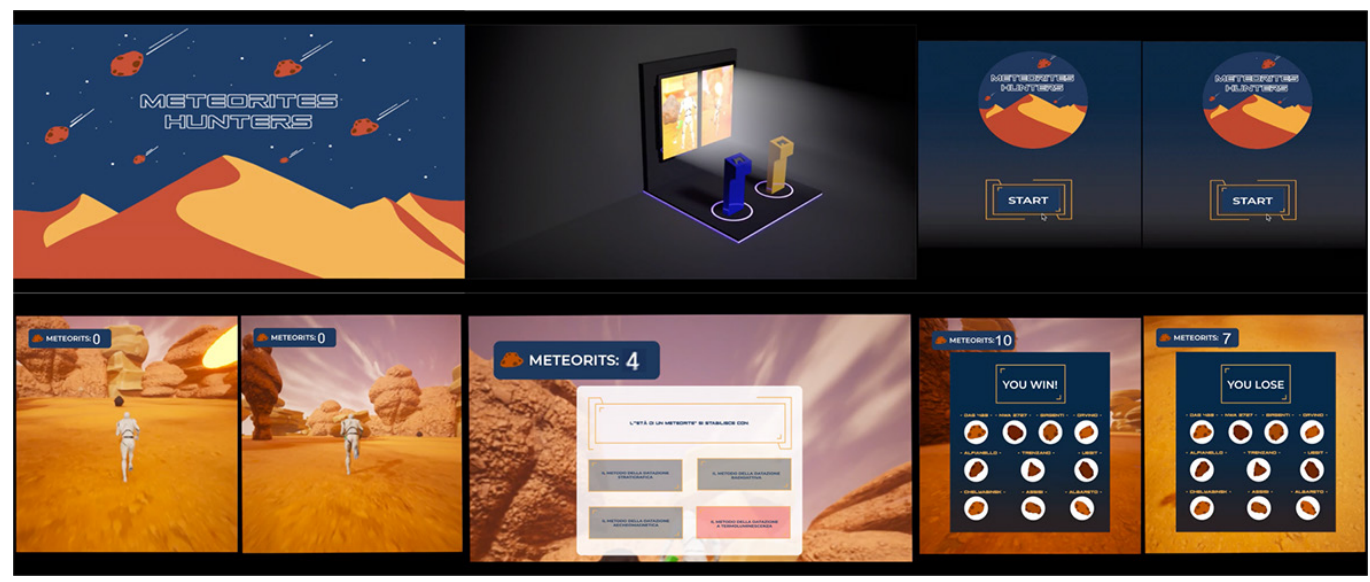

Se tradizionalmente il museo si basava su una comunicazione unilaterale, in cui mezzo e linguaggio (generalmente scelti dal depositario del dato scientifico) trasmettevano informazioni a un utente che le acquisiva passivamente, oggi si può definire il museo come 'transmediale': gli utenti diventano protagonisti di un'esperienza narrativa, interagiscono con le installazioni utilizzando i cinque sensi (vedere, toccare, muoversi, in alcuni casi odorare) e, grazie anche ai sistemi di iper-visualizzazione (realtà virtuale e aumentata), gli oggetti possono essere esplorati attraverso differenti strumenti. I percorsi di visita seguono schemi preordinati, ma i visitatori possono personalizzare la propria esperienza in base ai propri interessi ed i tempi di visita risultano dilatati rispetto alle visite nei musei tradizionali.

I musei transmediali utilizzano dunque strumenti di comunicazione che si rivelano di grande interesse per il settore del Disegno, poiché alla base ci sono forme di rappresentazione statiche, dinamiche, di iper-visualizzazione, che rendono la comunicazione dei musei più orientata alle esigenze ed aspettative dei visitatori.

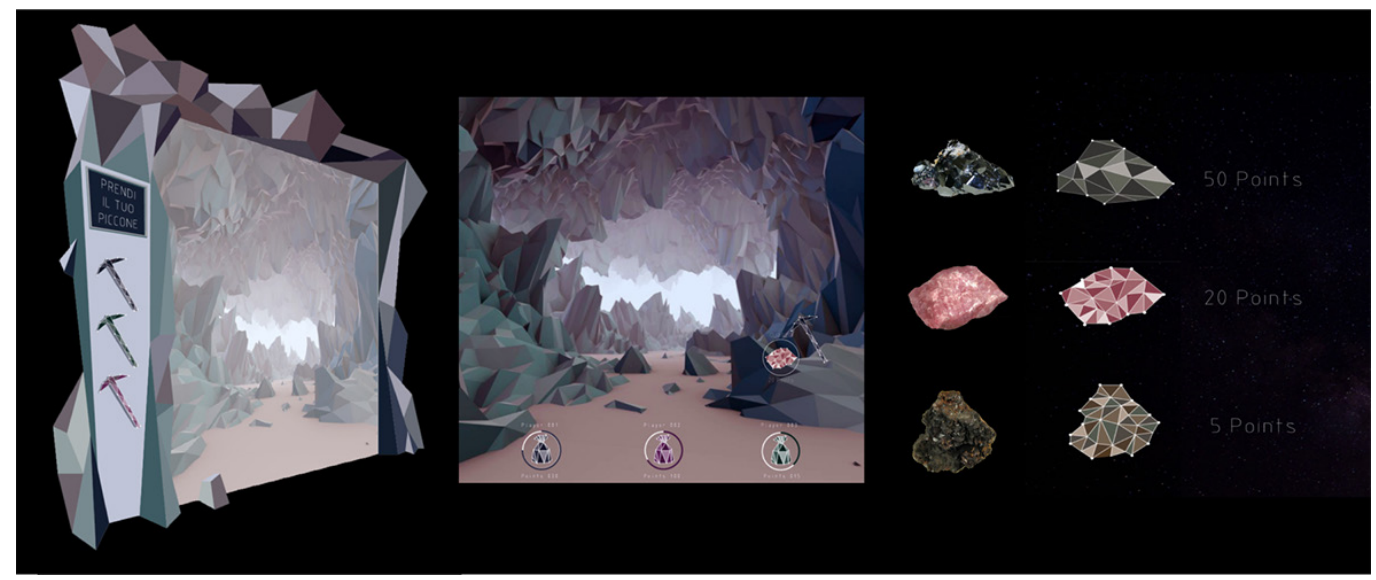

Fig. 3. Trova i Minerali è un'esperienza immersiva, interattiva e di serious game. Simula un ambiente minerario reale attraverso elementi materici in cui sono nascoste le tecnologie di gioco (una console wii, diversi display i controller com camuffati da picconi). II gioco consiste nel picconare pareti virtualmente simulate della miniera per trovare i minerali, di cui verranno for nite alcune informazioni al momento del ritrovamento. Quest'ultimo permette di accumulare punti che possono pol essere convertiti in sconti nello shop del museo (studenti: Villari, Gospodinova). 


\section{Stato dell'arte}

I musei multimediali sono di recente istituzione e solo in Italia ve ne sono almeno due di grande rilevanza: il Museo del '900 di Mestre (M9) ed il Museo Italiano Audiovisivo e Cinema (miac) di Roma. A questi si possono aggiungere i musei virtuali, che risiedono nel web, dove il visitatore naviga spazi immateriali in cui sono custodite repliche virtuali di architetture od opere d'arte, dove realtà e finzione si fondono e confondono lo stesso visitatore. Tra questi il Musée d'Art Contemporain VR 3D - Art Total Multimedia (macvr3d.com) o l'UMA (Universal Museum of Art) (the-uma.org). In questa direzione va anche la comunicazione dei cantieri di alcuni siti d'interesse storico, come il restauro del Mausoleo di Augusto a Roma, per il quale è stato allestito un sito che riguarda la 'Storia interattiva del Mausoleo di Augusto' (mausoleodiaugusto.it).

Alcuni musei 'transmediali' presenti nel panorama nazionale ed internazionale utilizzano strumenti di comunicazione legati all'edutainment, come il Museo della Citè des Sciences et de l'Industrie di Parigi, il Natural History Museum di Londra, oppure il Museo di Ötzi a Bolzano.

Fig. 4. Pink Beach concentra l'attenzione sulle spiagge da un punto di vista fossile e minerario.Viene ricreato un minerario. Viene ricreato un
ambiente immersivo totalambiente immersivo total-
mente virtuale, esplorabile attraverso l'uso di visori collegati ad un tapis roulant omnidirezionale che permette all'utente di muoversi e simulare parallelamente la locomozione nell'ambiente virtuale. (studenti: Ginnetti, Marino).

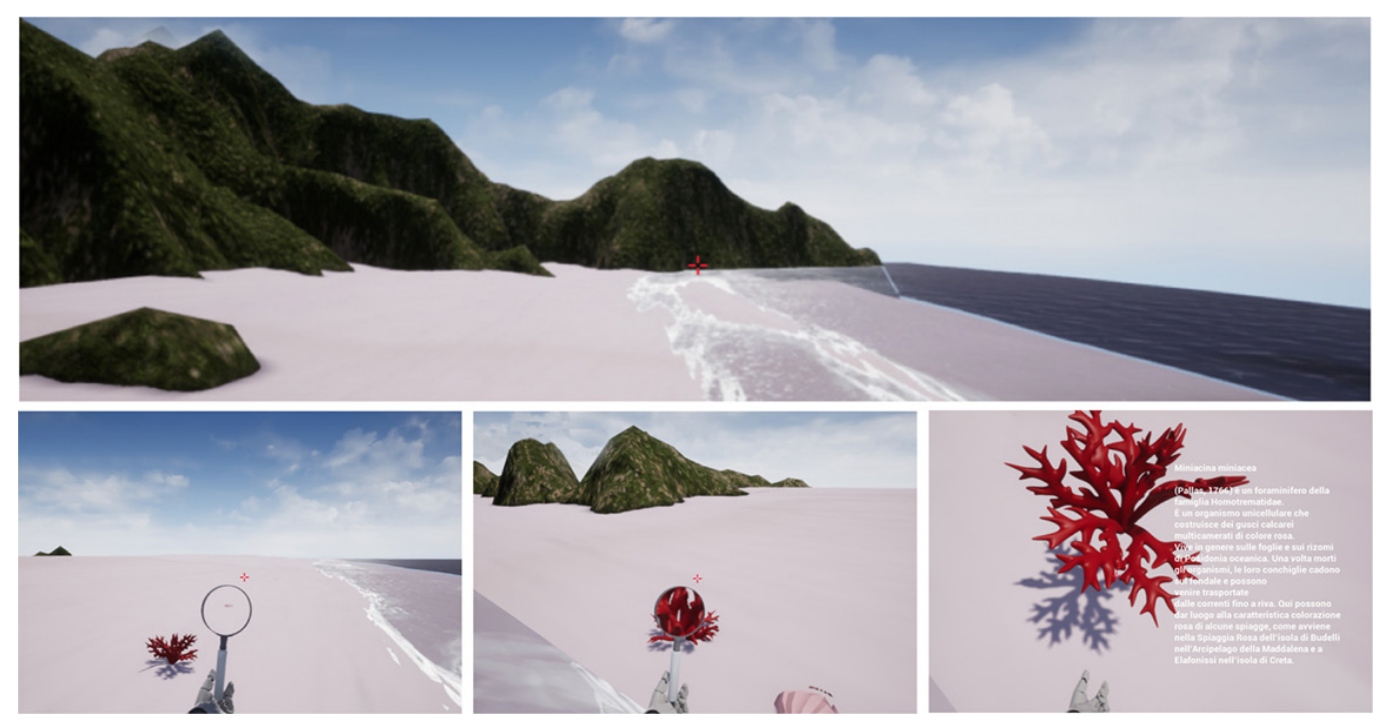

II Museo della Citè des Sciences et de l'Industrie di Parigi presenta esposizioni collegate alla diffusione delle scienze e delle tecniche nel mondo d'oggi. All'interno vi sono diverse mostre dove, al fianco degli oggetti esposti nelle tradizionali vetrine, si trovano modalità espositive collegate all'interactive experience di tipo analogico e digitale e all'immersive experience, come ad esempio nella sezione della 'Grande storia dell'universo' o il 'Foro stenopeico, rappresentazione dello spazio' in cui sono presenti spazi con illusioni ottiche come la camera di Ames, anamorfosi, e oggetti impossibili come in triangolo di 'Penrose'.

Anche nel Natural History Museum di Londra, interactive e immersive experience sono collocate al fianco della tradizionale esposizione per oggetti, con ricostruzioni di scheletri di dinosauri, simulatori di terremoto e olografie collocate a pochi metri di distanza gli uni dagli altri. In tutti questi musei, alla rappresentazione, nelle sue varie forme, viene attribuito un importante ruolo comunicativo, per far provare e comprendere anche fenomeni e soluzioni che la scienza non è ancora riuscita a rendere effettivamente realizzabili e replicabili (ad esempio lo strumento dell'olografia, che seppur percepibile con fenomeni collegati all'illusione ottica, è ancora relegato all'ambito del fantasy e della fantascienza ed è grazie agli studi sulla riflessione e la visualizzazione che è possibile realizzarlo). 


\section{Metodologia della ricerca}

La ricerca si focalizza sulle modalità di rappresentazione che, attraverso gli strumenti messi a disposizione dall'industria dell'edutainment, divengono linguaggi di comunicazione e divulgazione. L'edutainment, crasi delle parole anglosassoni education (educazione) ed entertainment (intrattenimento), utilizza diverse modalità di comunicazione del tutto coerenti con il ruolo del museo transmediale e le nuove modalità di accesso culturale: interactive experience, immersive experience, serious game e gamification, universal design.

Queste modalità non agiscono separatamente le une dalle altre, ma presentano sovrapposizioni continue che creano un'esperienza emotiva la quale favorisce i processi cognitivi e dunque l'apprendimento. Tutte le categorie hanno una preponderante componente legata alla rappresentazione 3D, alle ricostruzioni virtuali e al visual, che, uniti allo storytelling, contribuiscono a migliorare l'acquisizione delle informazioni.

I casi studio presentati sono frutto di una ricerca svolta dagli autori in ambito didattico con i ragazzi dei corsi della Facoltà di Architettura, Sapienza Università di Roma [I] che hanno elaborato delle proposte per il nuovo Museo Universitario di Scienze della Terra (MUST) del Polo Museale Sapienza.

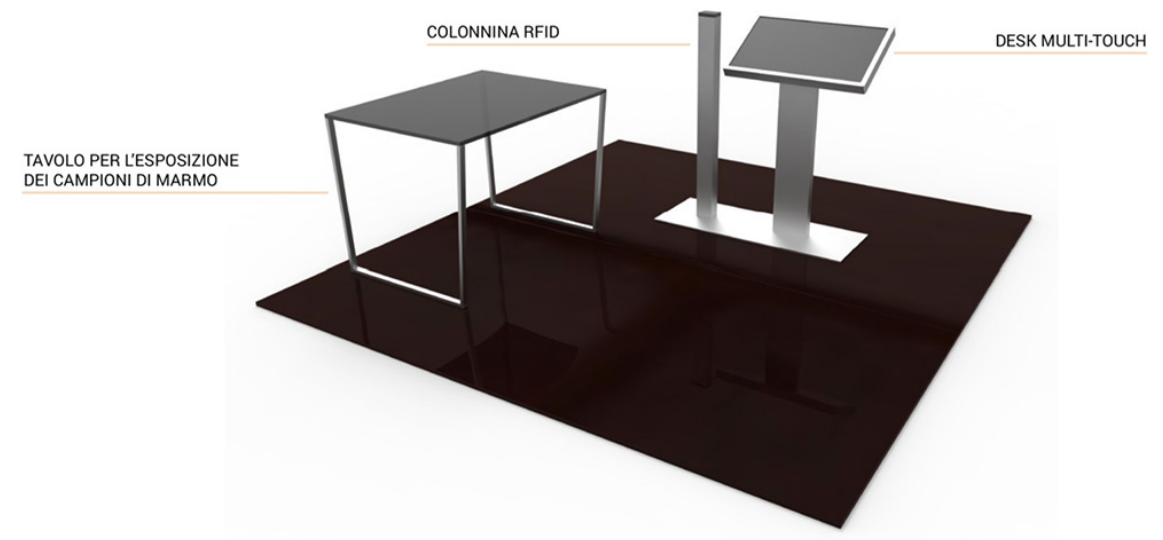

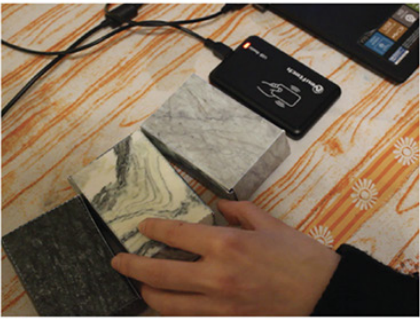

SCELTA DEL MARMO

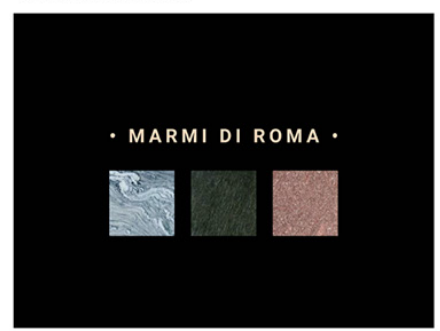

SCHERMATA INIZIALE DEL TOUCH SCREEN RFID INATTIVO

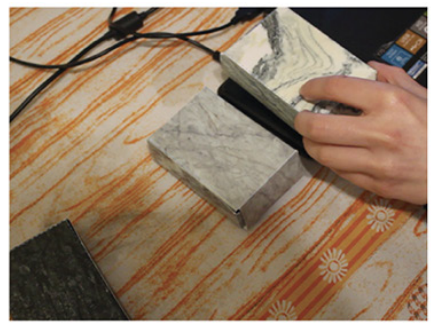

POSIZIONAMENTO SU LETTORE RFID

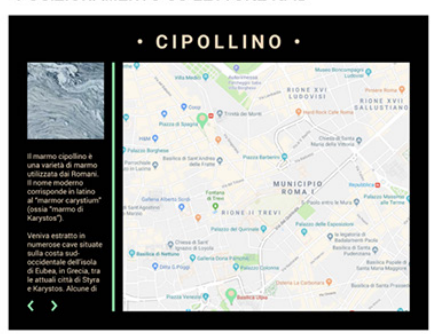

LETTURA DEL SEGNALE INVIATO DAL DISPOSITIVO SELEZIONE DEL SITO DI INTERESSE RFID E RICONOSCIMENTO DEL MARMO

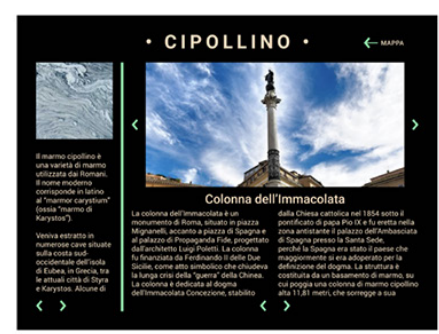

Fig. 5. Vista tridimensionale e, sotto, i diversi passaggi Barillari, Paoli). 


\section{Interactive Experience}

L'esperienza interattiva, che prevede un reciproco scambio di input e feedback tra utente e macchina, si divide in due categorie: le esperienze analogiche che si basano sull'emissione di un input da parte dell'utente attraverso il contatto diretto con la macchina (girare, premere un pulsante, abbassare o alzare una leva ecc.); le interazioni digitali rilevano i movimenti dell'utente mediante dispositivi che trasformano un input in azioni 'macchina' e non prevedono un contatto diretto con l'installazione. Sono utilizzati strumenti nativi dell'ambito del gaming, spesso basati sul riconoscimento gestuale quali leap motion 3D, microsoft Kinect, Nintendo wii ecc. Ai fini di un'efficace interattività, in cui i tempi di risposta e la velocità di esecuzione della macchina sono fondamentali, la rappresentazione grafica deve presentare un giusto equilibrio tra numero di poligoni degli elementi modellati, capacità di visualizzazione e velocità di processo degli input ricevuti dall'utente.

L'installazione I Marmi di Roma (Barillari, Paoli, DCVM I8-19) sfrutta la modalità interattiva analogica per rivoluzionare il modello espositivo del campionario di marmi presenti nella sezione di Geologia del MUST Sapienza, che allo stato attuale si presenta come una bacheca al cui interno sono esposti i marmi insieme ad una targa identificativa e ad un'immagine di opere/edifici/monumenti in cui quel marmo è presente. Attraverso un sistema di riconoscimento RFID applicato sui campioni e uno schermo touch, l'utente può scegliere un tipo di marmo ed esplorarne le caratteristiche 'geologiche' oltre che navigare una mappa di Roma con la possibilità di approfondire le modalità di utilizzo del marmo scelto in determinate opere o edifici.

Fig. 6. Storyboard che illustra le fasi dell'esperienza in Augmented

Space (studenti: Carosi

Mirenda).
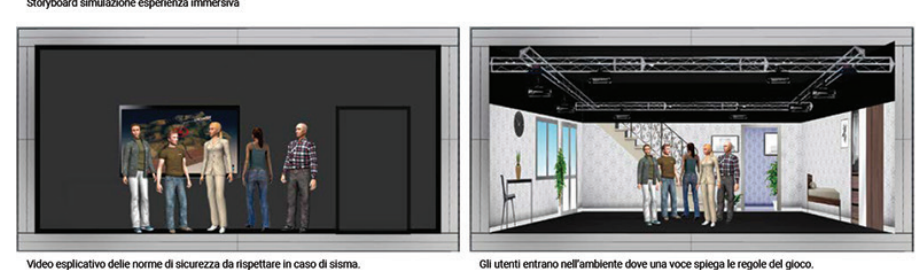

Gill utent entrano neframblente dove una voce splega le regole ded gloco.
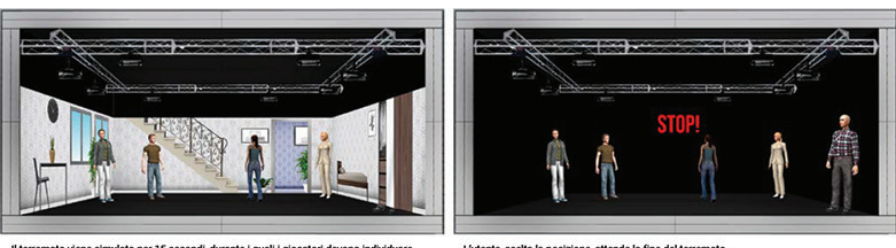

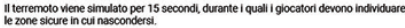

Lutente, sceta la possizone, attende la fine del teremoto
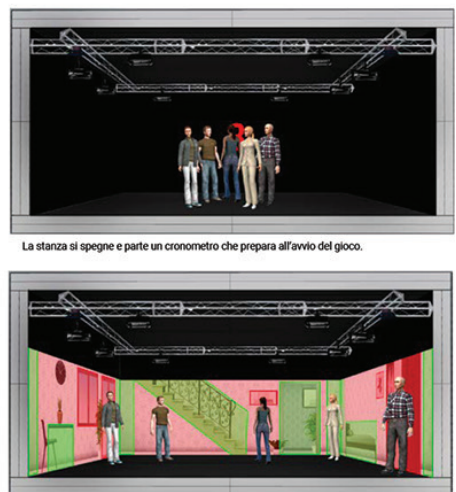

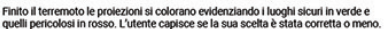

\section{Immersive Experience}

Un'esperienza immersiva è una particolare modalità di fruizione di contenuti in cui l'osservatore è totalmente immerso in uno spazio costruito o ricostruito virtualmente con cui può interagire. II termine 'immersione' si riferisce, dal punto di vista percettivo, alla capacità del suddetto spazio di coinvolgere direttamente i sensi del soggetto, isolandolo dagli stimoli dell'ambiente reale. I sistemi immersivi in Augmented Reality (AR), attraverso l'uso di strumenti smart dotati di sistemi GPS e AR 'tracking', consentono di sovrapporre reale e virtuale e permettono all'utente di agire in real time acquisendo più tipologie di informazioni [Azuma 1997]. II sistema di Virtual Reality (VR) simula invece uno spazio in cui l'utilizzatore ha la percezione di trovarsi e potersi muovere all'interno interagendo con gli oggetti presenti. A questi si aggiunge l'Augmented Space [Maniello 20 I 8], che si origina dalla sovrapposizione di livelli informativi derivanti da dati informatici all'interno di uno spazio reale. Ciò si traduce nell'allestimento di stanze immersive in cui, attraverso proiezioni, si arricchisce lo spazio reale di contenuti ed informazioni. 
Earthquake Room (Carosi, Mirenda, DCVM I8/19) è un progetto che vuole educare le persone sia sul fenomeno naturale del terremoto, sia al comportamento da adottare in caso di evento sismico. L'esperienza è progettata secondo la tecnologia dell'Augmented Space: l'utente si trova all'interno di una stanza arredata virtualmente tramite proiettori e, durante il tempo di simulazione del sisma, deve riuscire a posizionarsi in corrispondenza dei posti ritenuti sicuri secondo le norme di sicurezza sulle quali sarà stato precedentemente informato. A fine simulazione, la cui durata prevista è di 15 secondi, le proiezioni sul muro indicheranno quali sono le posizioni sbagliate (in rosso), e quali quelle corrette (in verde).

Fig. 7.Immagini delle proiezioni di due delle siva: lo stato delle pareti all'inizio del gioco; la parte bassa illustra tra, la parte bassa illustra, tramite la colorazione verde e rossa, i punti sicuri e di pericolo che si attivano a gioco

concluso (studenti: Carosi, Mirenda).
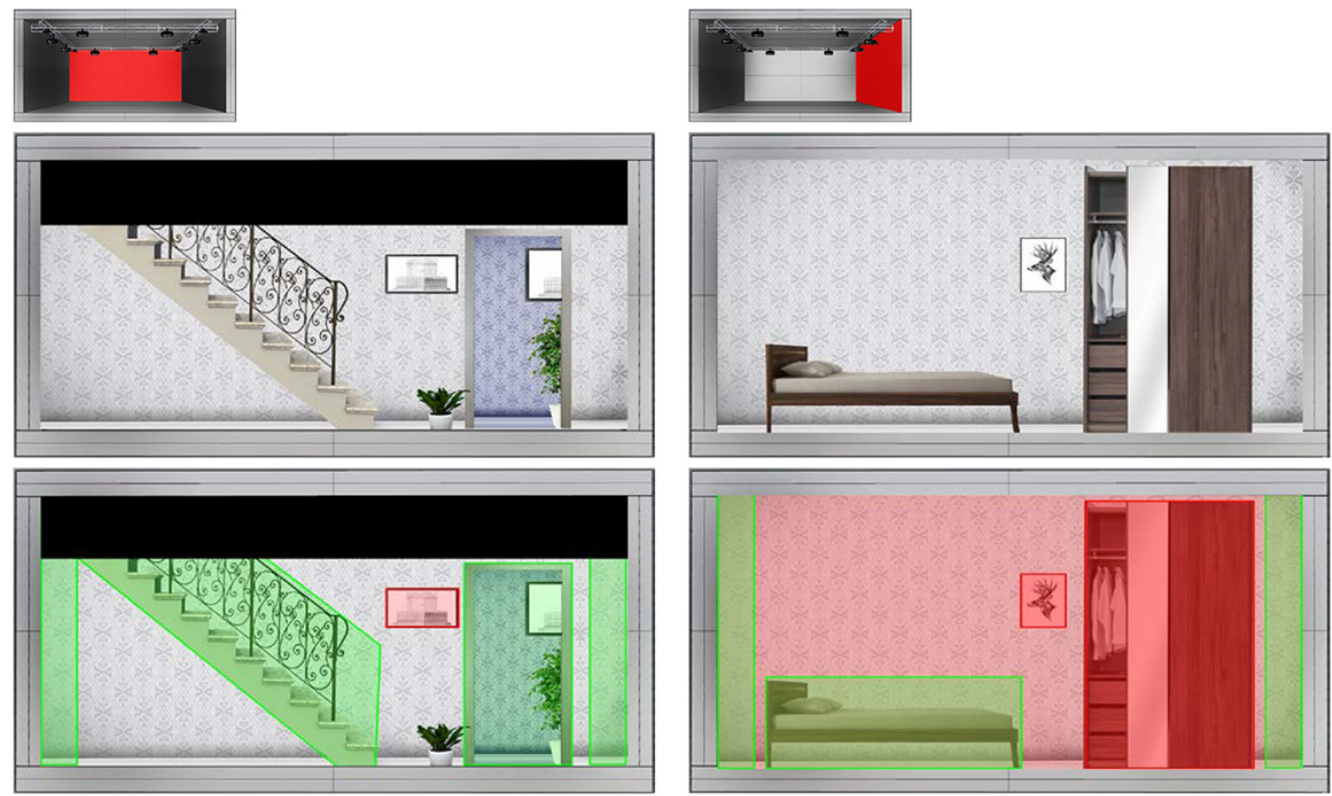

\section{Serious Game e Gamification}

I serious game provengono direttamente dal settore dei videogiochi, ma nascono a scopo educativo. Sono giochi 'simulativi' (cioè che riproducono fedelmente alcuni aspetti del mondo reale) che, attraverso la risoluzione di enigmi o di situazioni, stimolano l'apprendimento ed aumentano l'accessibilità culturale ampliando l'età dell'utenza.

I sistemi di gamification risultano essere molto utili nell'esplorazione del percorso museale. In questo caso non si tratta di 'creare un gioco' ma di sperimentare schemi e meccanismi legati ai videogiochi per spingere gli utenti a compiere alcune azioni, premiandoli in maniera intrinseca o estrinseca. Traendo vantaggio dall'apprendimento interattivo, la gamification rappresenta uno strumento efficace per veicolare un messaggio e indurre l'utenza ad avere comportamenti attivi.

II serious game interattivo Rock Seeker (Persiani, Milan, DCVM I8/19) racconta da un particolare punto di vista la Dattilioteca, preziosa raccolta di anelli appartenenti a Papa Leone XII costituiti da pietre preziose, esposta al MUST Sapienza. II gioco permette l'esplorazione di una miniera virtuale in cui l'utente è invitato a cercare le diverse pietre che compongono gli anelli papali. Una volta trovati l'utente può approfondire le informazioni sulle pietre che ha scoperto. L'interazione avviene in una stanza 'immersiva' in cui 3 pareti riproducono analogicamente l'ambiente di gioco, mentre nella quarta viene proiettato il gioco, utilizzabile con un classico controller. 


\section{Universal Design}

L'Universal Design può intendersi come modalità comunicativa che cerca di garantire la piena fruibilità delle esposizioni a tutti i potenziali visitatori consentendo una maggiore accessibilità culturale [Empler 20।8]. È legata all'eliminazione delle barriere architettoniche (intese sia fisiche che sensoriali), ed ha sia una componente progettuale che una di rappresentazione. Quest'ultima si lega al settore delle disabilità sensoriali e cognitive e comprende tutte quelle soluzioni per disabili visivi (che utilizzano il tatto come mezzo di comunicazione e per cui si deve strutturare un linguaggio visuo-tattile) [Empler, Fusinetti 2019], uditivi (che fanno della vista il canale privilegiato) [Empler 20 I3] e cognitivi, utilizzate per semplificare la comunicazione. Le soluzioni devono avere una comunicatività tale da rendere immediatamente riconoscibili e comprensibili messaggi anche piuttosto complessi.

L'iSense Room è un'installazione pensata in modo accessibile, parte introduttiva della mostra temporanea del MUST. La stanza, di forma quadrata, ha agli angoli 4 piedistalli su cui sono disposti dei minerali con cui è possibile interagire tramite olfatto (calcare bituminoso), tatto (quarzo) e gusto (salgemma, che viene fornito sotto forma di bustine di sale da cucina). Una postazione centrale, protetta, contiene minerali sensibili ai raggi UVA che vengono alternatamente illuminati per mostrare come cambia il loro colore al variare della tipologia di luce. L'installazione è immersiva, il visitatore si ritrova in una stanza buia e viene guidato da una voce narrante che, attraverso l'ausilio di una doppia proiezione video su pareti (predisposta con supporto LiS per i non udenti), lo accompagna nell'esplorazione del pianeta Terra attraverso i 5 sensi.

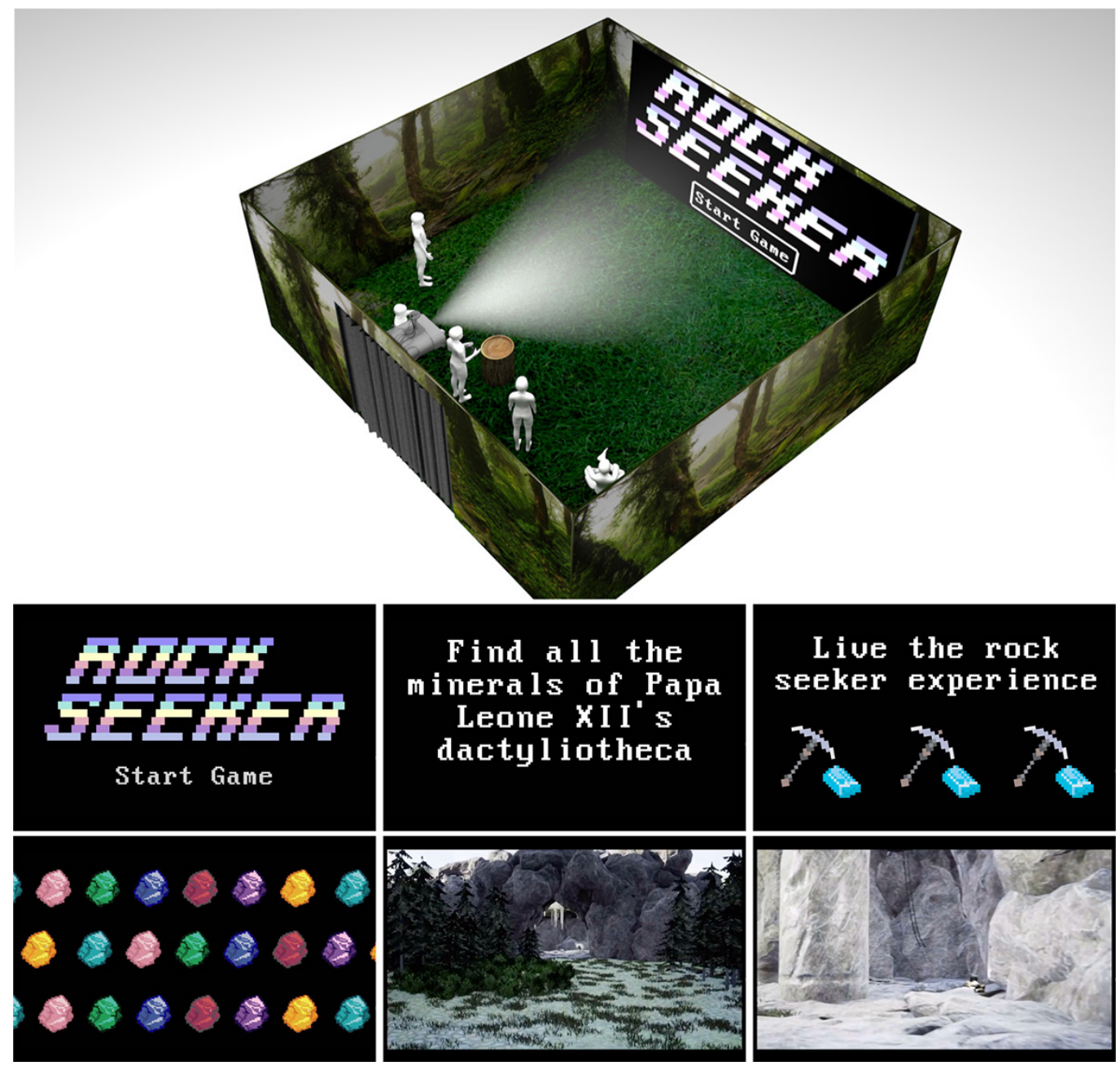


Fig. 9. Schema compositivo dell'iSense Room in cui sono evidenziate le interazioni con i 5 sensi (studenti: Neroni, Papi).

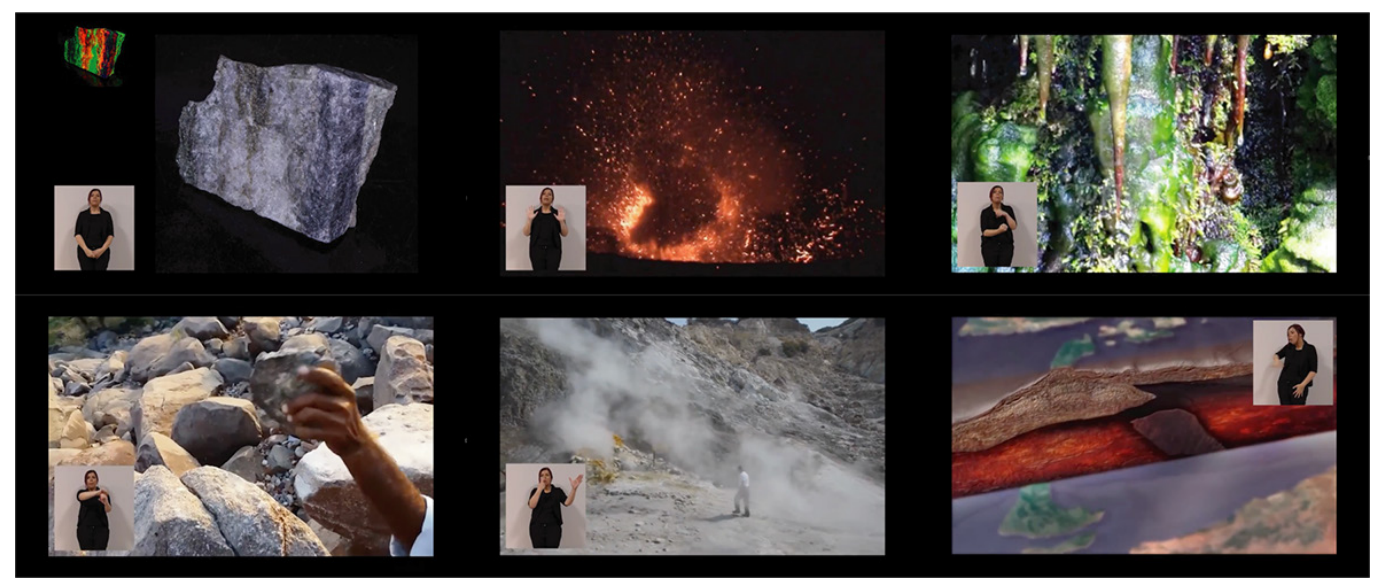

\section{Conclusioni}

II museo 'transmediale' designa un approccio complesso alla comunicazione e all'accesso culturale, e denota una crescente attenzione da parte di discipline differenti (dalla rappresentazione, alla sociologia, alla semiotica, ai new media studies) che assumono una prospettiva trasversale al fine di coinvolgere un più grande numero di utenti. Tutti i linguaggi e gli strumenti descritti nella metodologia adottata, non sono da considerarsi quali settori separati, ma interconnessi tra loro con diverse sovrapposizioni col fine di incrementare l'offerta di servizi di assistenza culturale attraverso apparati comunicativi che trasmettano efficacemente i valori del patrimonio. Se i curatori dei musei rispondono alla domanda 'cosa comunicare', la rappresentazione delle informazioni, risponde alle domande 'come comunicare' e 'a chi comunicare', assumendosi il ruolo di connettore tra l'apprendimento, le conoscenze dei visitatori, con i saperi specialistici. Queste strategie, insieme ad una spiccata attenzione verso accesso, partecipazione e rappresentazione, stimolano curiosità, interessi e motivazioni durante il percorso museale, attivando processi partecipativi che permettono di vivere pienamente l'esperienza museale.

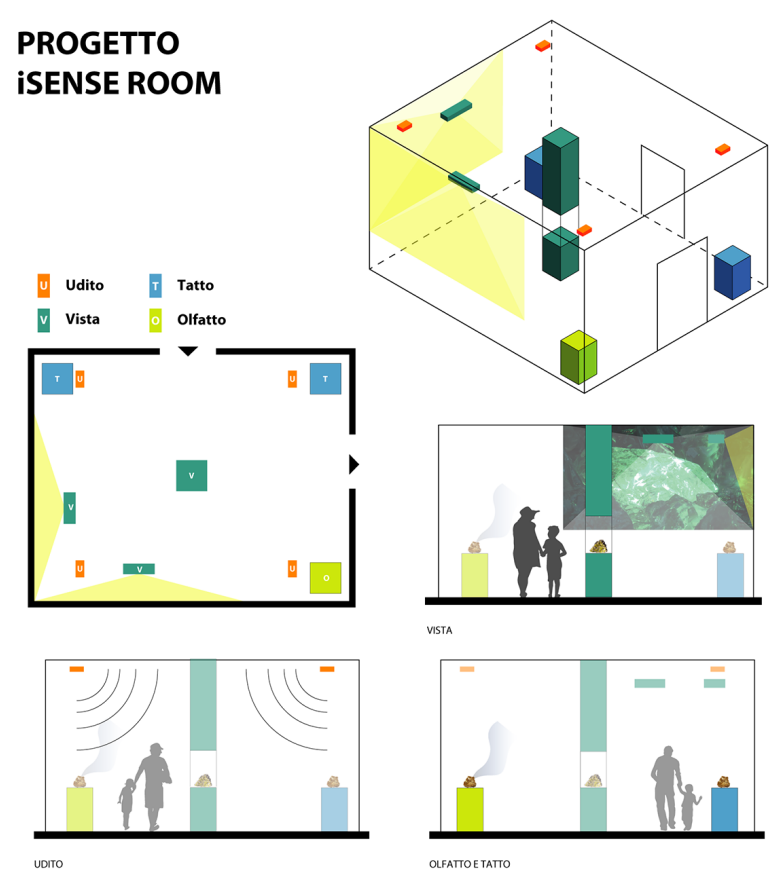




\section{Note}

[I] Corso di Laurea Magistrale in Design Comunicazione Visiva e Multimediale, Corsi di Computer Grafica e Multimedia Design I (corso sviluppato in collaborazione con Lauren Ferro, Dipartimento di Ingegneria informatica, automatica e gestionale, Sapienza Università di Roma).

\section{Riferimenti bibliografici}

Antinucci Francesco (20/4). Comunicare nel museo. Bari: Laterza.

Azuma Ronald T. ( 1 997). A survey of augmented reality. In Presence:Teleoperators and Virtual Environment 6(4), pp. $355-385$.

Da Milano Cristina, Sciacchitano Erminia (20I5). Linee guida per la comunicazione nei musei: segnaletica interna, didascalie e pannelli. In Guido Manuel R. (a cura di). Quaderni della valorizzazione - NS I. Roma: Ministero dei Beni e delle Attività Culturali e del Turismo - Direzione Generale dei Musei - Servizio II - Gestione e valorizzazione dei musei e dei luoghi della cultura.

Empler Tommaso (20 I3). Universal Design: ruolo del Disegno e Rilievo. In Disegnare, Idee, Immagini, n. 46, pp......

Empler Tommaso (20I8). Traditional Museums, virtual Museums. Dissemination role of ICTs. In DisegnareCon. Advanced Technologies for Historical Cities Visualization, vol. I I, n. 2 I, p.......

Empler Tommaso, Fusinetti Alexandra (2019). Rappresentazione visuo-tattile. Comunicazione tattile per i disabili visivi. In Belardi Paolo (a cura di). Riflessioni, l'arte del disegno/il disegno dell'arte. Atti del $41^{\circ}$ Convegno Docenti delle discipline della rappresentazione, Perugia 19-2I settembre 2019. Roma: Gangemi Editore.

Jenkins Henry (20|4). Cultura convergente. Rimini: Maggioli editore.

Maniello Donato (20।8). Tecniche avanzate di video mapping. Spatial Augmented Reality applicata al bene culturale. Brienza: Le Penseur.

\section{Sitografia}

Macvr3D.com, disposibile su: <https://macvr3d.com/>.

Mausoleodiaugusto.it, disponibile su: <http://www.mausoleodiaugusto.it/it/>.

The-uma.org, disponibile su: <https://the-uma.org/>.

Treccani, disponibile su: <http://www.treccani.it/enciclopedia/transmedia-storytelling_(Lessico-del-XXI-Secolo)/>.

\section{Autori}

Tommaso Empler, Sapienza Università di Roma, tommaso.empler@uniromal

Adriana Caldarone, Sapienza Università di Roma, adriana.caldarone@uniromal.it

Alexandra Fusinetti, Sapienza Università di Roma, alexandra.fusinetti@uniromal.it

Per citare questo capitolo: Empler Tommaso, Caldarone Adriana, Fusinetti Alexandra (2020). Musei tra narrazione, visualità e new media. Museums between narration, visuality and new media. In Arena A., Arena M., Brandolino R.G., Colistra D., Ginex G., Mediati D., Nucifora S., Raffa P. (a cura di). Connettere. Un disegno per annodare e tessere. Atti del $42^{\circ}$ Convegno Internazionale dei Docenti delle Discipline della Rappresentazione/Connecting. Drawing for weaving relationships. Proceedings of the 42 th International Conference of Representation Disciplines Teachers. Milano: FrancoAngeli, pp. 3293-3312. 


\title{
Museums between Narration, Visuality and New Media
}

\author{
Tommaso Empler \\ Adriana Caldarone \\ Alexandra Fusinetti
}

Abstract

IAnalyzing museum's communication scenery, we can find two extremes: a traditional museum, a real space in which the visitor passively acquires selected information through a pre-chosen language, and a multimedia museum, a virtual space designed to make the visitor play an active and participatory role, letting him choose his own visit path and select independently the information he wants to acquire.

In between there is a particular type of museum that can be defined as "transmedia" with communicative tools and languages that transform learning processes and levels of attention. These tools and languages refer to the field of edutainment and they are: interactive experience, immersive experience, serious games, gamification and universal design. In these museum emitter and receiver are connected by mutual interrelationships and correlations.

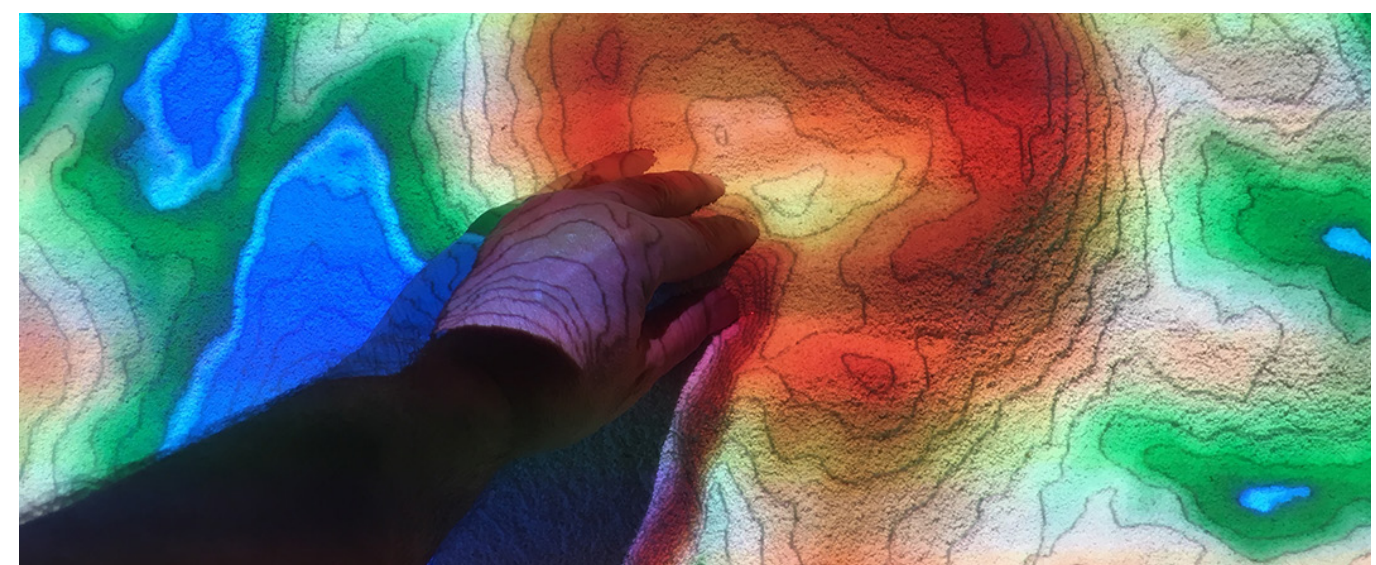




\section{Introduction}

Museography today goes from eighteenth-century traditional museums' organization, when the discipline was born, to the recent virtual museums.

Traditional museums were initially structured according to the Wunderkammer logic, rooms of wonders in which extraordinary objects were collected. Nowadays 'museum' concept, both according to what is set out in "Guidelines for communication in museums" [Da Milano, Sciacchitano 20 I5], and to the new definition proposed by ICOM, is associated with the diffusion of knowledge and the educational role that derives from it. Director or curator are the museum's information keepers, who often compare themselves with visual designers to choose correct form of representation that rightly conveys message and knowledge. Since in exhibitions objects are extrapolated from their context of origin, useful for their correct semantic interpretation, it is necessary that communication systems supports the objects comprehension [Antinucci, 20 I4]. An effective method that museum communication field uses today is storytelling, a technique that consists in telling a story seeking the involvement and attention of the public to convey the message.

Fig. I. Sulle Orme della Conoscenza is an interactive and serious game installation in which, startin from the fossils of the reptile's footprints, we want to explain howe formed and what infor mation they can prove mation they can provide regarding the physical and behavioral characteristics of the animal.Through holographic projections and motion sensors, game moments will alternate with technica explanations (students: Candida, Ferrante).
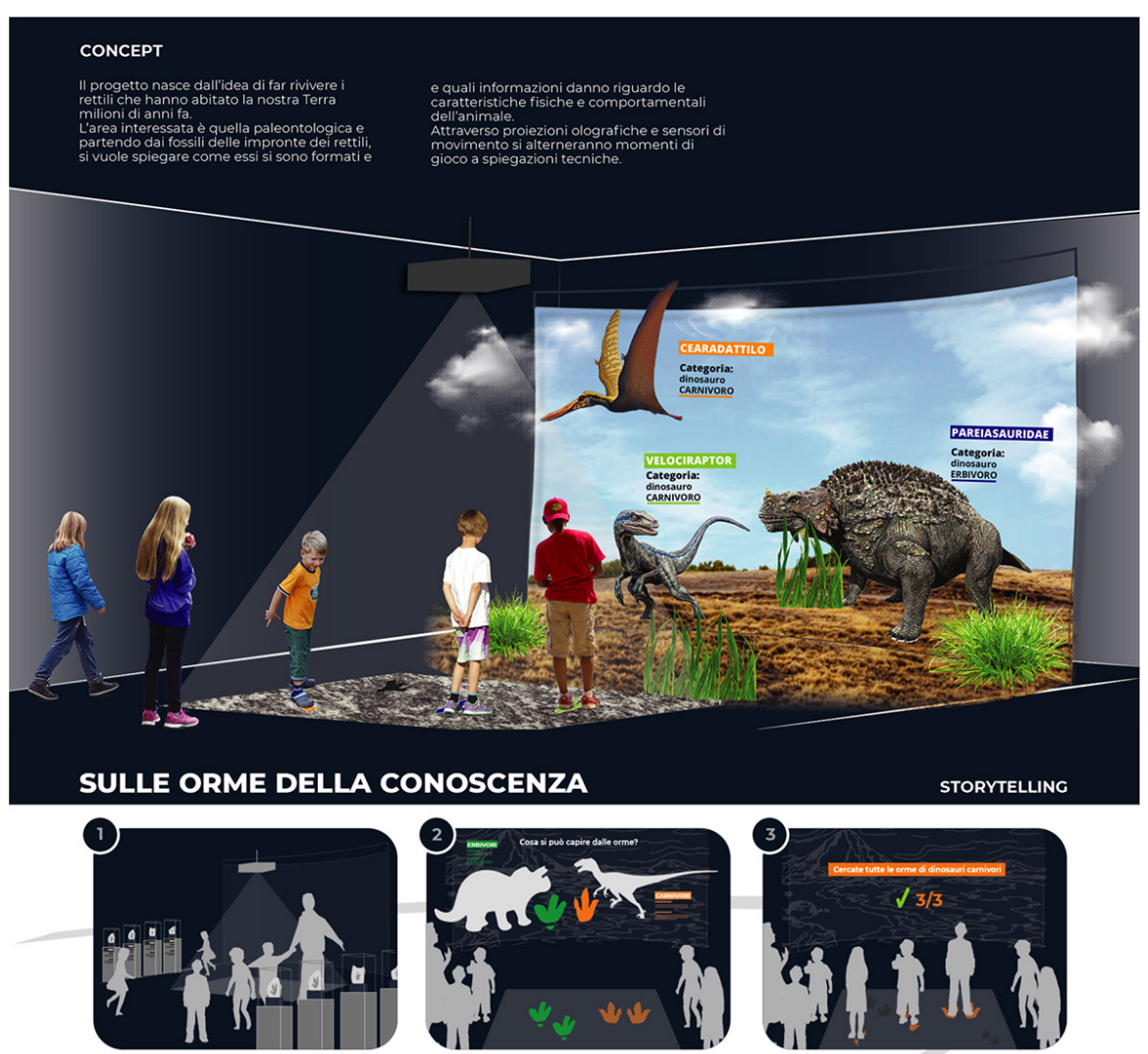

Massimo 10 persone entrano nella stanzea si posizionano intorno al
proiezione a terra. Interno a loro Ascoltano una introduzione da una
voce accompaganata da immagini.

Spiegazione di cosa si può capire che con il tempo sono diventate icre tresclin Una volta finita la spiegazione vengo no proposti ar bambini una serie di
giochi per riconoscere le varie orme secondo quanto ascoltato. (es. ricon
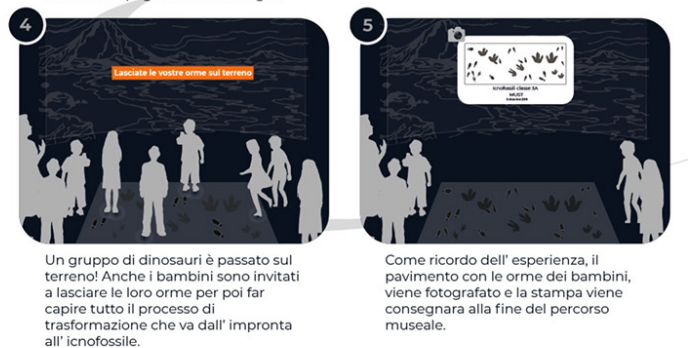

Come ricordo dell' esperienza, il pavimento conle orme dei bambini,
viene fotografato e la stampa viene consegnara alla fine del percorso

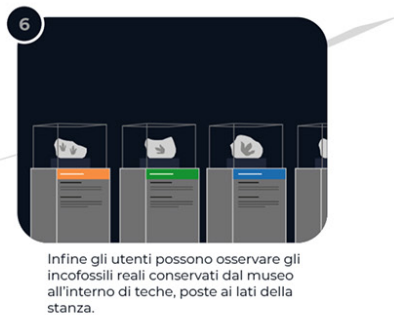


Technological evolution has defined a transmedia storytelling [Jenkins 20 I4]: a story is told on different media and platforms, in which every single tool or communication language offers a specific contribution to the entire narrative complex. It is not a question of repeating the main story on another media, but of entrusting part of the narration to various media, thus also involving different types of viewers [Treccani 2020, ad vocem].

Fig. 2. Serious game, Meteorite Hunters aims to inform and educate adults and children about the world of meteorites. As oroolo sts, players will be able to discover all the curiosities and and properties of meteorites precipitated on earth, searching for real samples in a desert environment. Each stone will be kept in a virtual backpack, the more meteorites will be found, the higher the score. Everything will be accompanied by a quiz to which players will have to answer to continue the game and get the meteorites (students: Pignatone Laurini, Pilato).

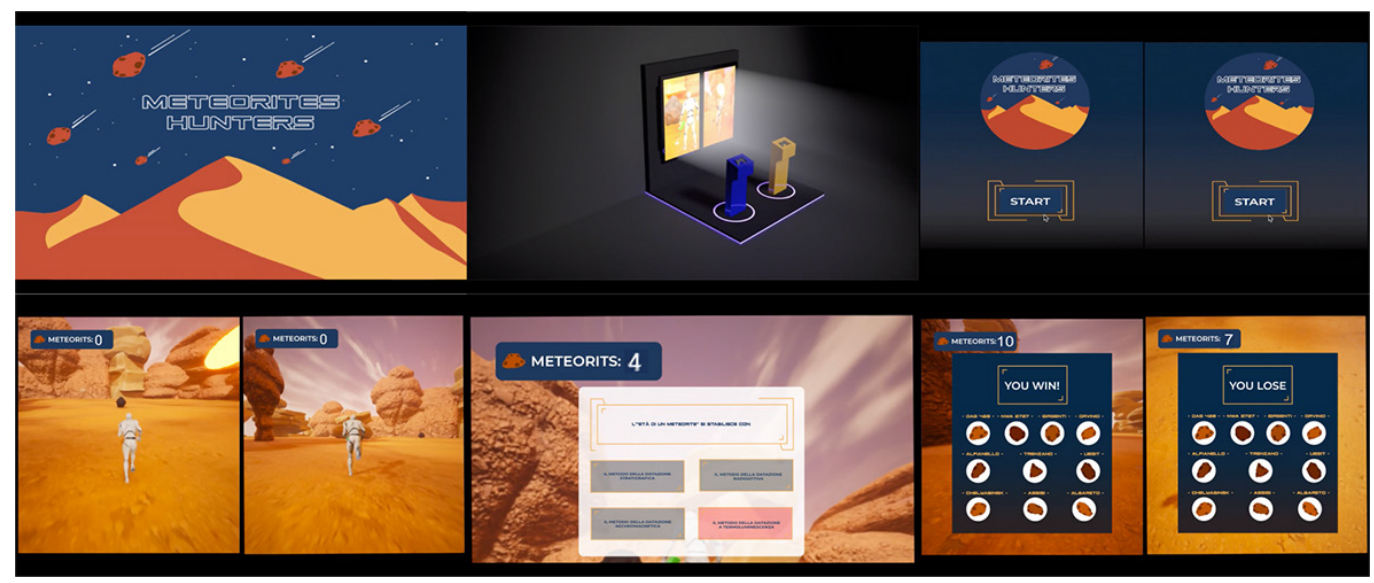

If traditionally museums was based on unilateral communication, in which medium and language (generally chosen by the depositary of scientific data) transmitted information to a user who acquired it passively, today museums can be defined as 'transmedia': users become protagonists of a narrative experience, they interact with the installations using five senses (seeing, touching, moving, in some cases smelling), hyper-visualization systems (such virtual and augmented reality), and the objects can be explored through different tools. Visit itineraries follow preordained schemes, but visitors can customize their experience according to their interests; visit times are therefore dilated compared to visits to traditional museums. Transmedia museums thus use communicative tools which prove to be of great interest for the sector of representation, since we can find forms of static, dynamic, hyper-visualization, which make museum communication more oriented to the needs and expectations of visitors.

Fig. 3. Trova i Minerali is an immersive, interactive and serious game experience. It simulates a real mining environment designed through materia elements in which game elentid technologies are hidden (a Nintendo Wil console several displays and controllers disguised as pickaxes). Game consist in "picking" the virtually simulated walls of the mine to find minerals, of which some information will be provided at the time of the discovery. Also, discovery allows you to accumulate points that can then be converted into discounts in the museum shop. (students:Villari, Gospodinova).

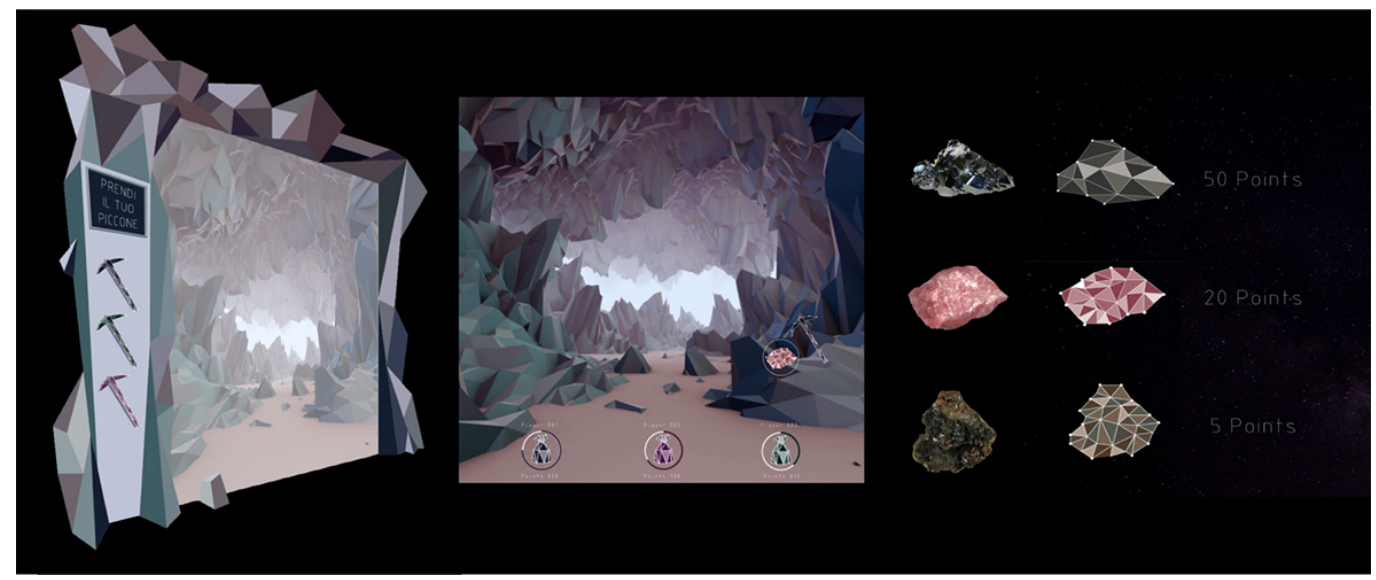




\section{State of art}

Multimedia museums are recently established and only in Italy there are at least two of great importance: Museum of '900 in Mestre (M9) and the Italian Audiovisual and Cinema Museum (miac) in Rome. Can be added to these virtual museums, of web residency, where visitors navigates intangible spaces in which are kept virtual replicas of architectures or works of art, where reality and fiction merge and confuse visitors, such as Musée d'Art Contemporain VR 3D - Art Total Multimedia (macvr3d.com) or UMA (Universal Museum of Art), (the-uma.org). Some historical places' construction sites has developed a communication system that convey some information about the object being restored, as it is happening around the Mausoleum of Augustus in Rome, for which was set up a website connected to the external protection of the construction site, made 'interactive' through images games, optical illusions, sounds, where the story of Augustus and his Mausoleum is told. (mausoleodiaugusto.it).

Some 'transmedia' museums, nationally and internationally, use communication tools related to edutainment, such as Museum of Citè des Sciences et de l'Industrie in Paris, Natural History Museum in London, or Ötzi Museum in Bolzano.

Fig. 4. Pink Beach focuses attention on the beaches from a fossil and mining point of view. A totally virtual immersive environment is recreated, which can be explored through the use of viewers connected to an omnidirectional treadmill that allows the user to move and simulate paralle locomotion in the vartul environment (students: Ginnetti, Marino).

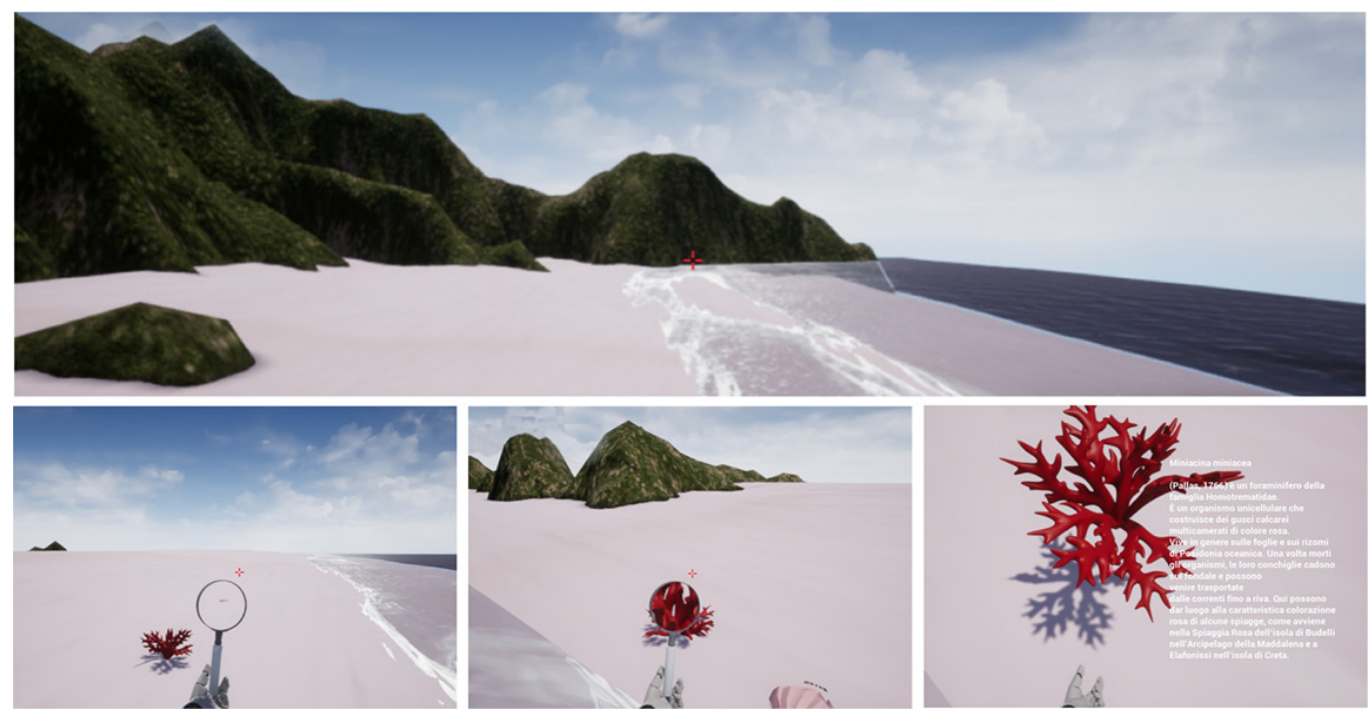

Museum of Citè des Sciences et de l'Industrie in Paris presents exhibits that narrates the diffusion of sciences and techniques in today's world. Inside there are several exhibitions where, alongside traditional showcases, are displayed analog/digital interactive experience or immersive ones, such as Ames' room optical illusion, anamorphosis and impossible objects such as in the 'Penrose' triangle, all located in the 'Great history of the universe' or the 'Pinhole, representation of space' sections.

Also in Natural History Museum in London, interactive and immersive experiences are placed alongside the traditional exhibition, with reconstructions of dinosaur skeletons, earthquake simulators and holographs located a few meters away from each other.

In all these museums, representation, in its various forms, has an important communicative role, among which to try and understand phenomena and solutions that science has not yet managed to actually make feasible and replicable, (for example holography tool, which, although perceptible with phenomena connected to optical illusion, is still relegated to the field of fantasy and science fiction and, thanks to studies on reflection and visualization, it is possible to achieve it). 


\section{Research methodology}

Our research focuses on representation methods which, through the tools made available by the edutainment industry, become languages of communication and dissemination. Edutainment, a word-combination of 'education' and 'entertainment', uses different communicative methods, coherent with the role of transmedia museum and new cultural access methods: interactive experience, immersive experience, serious game and gamification, universal design.

These modalities do not act separately from each other, but present continuous overlaps that create an emotional experience which favors cognitive processes and therefore learning. All categories have a predominant component linked to 3D representation, virtual reconstructions and visual communication, which, combined with storytelling, contribute to improving the acquisition of information.

Presented case studies are the result of research carried out by the authors in the educational field with students of Faculty of Architecture, Sapienza University of Rome [I] who have developed proposals for the new University Museum of Earth Sciences (MUST) of Polo Museale Sapienza.

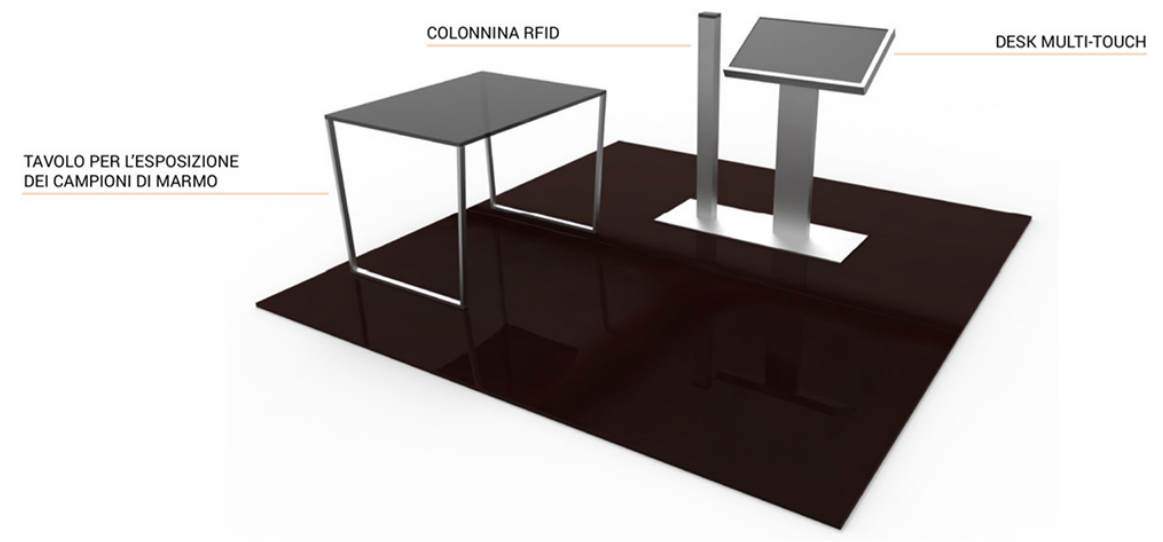

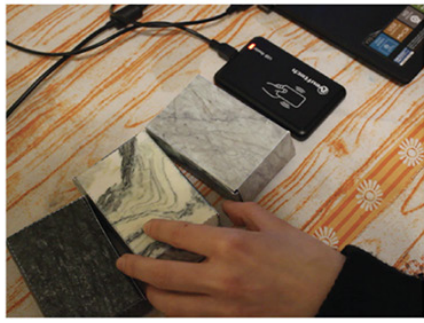

SCELTA DEL MARMO

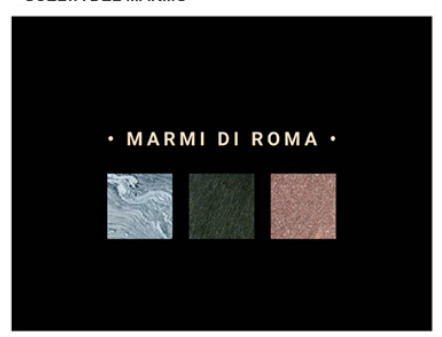

SCHERMATA INIZIALE DEL TOUCH SCREEN RFID INATTIVO

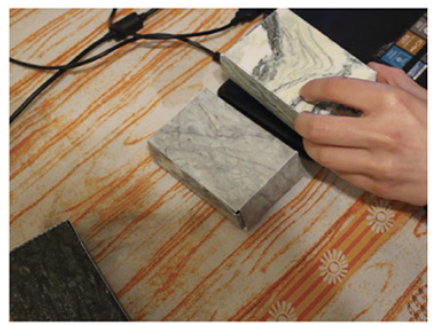

POSIZIONAMENTO SU LETTORE RFID

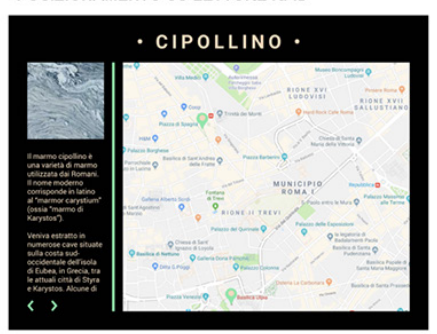

LETTURA DEL SEGNALE INVIATO DAL DISPOSITIVO SELEZIONE DEL SITO DI INTERESSE RFID E RICONOSCIMENTO DEL MARMO

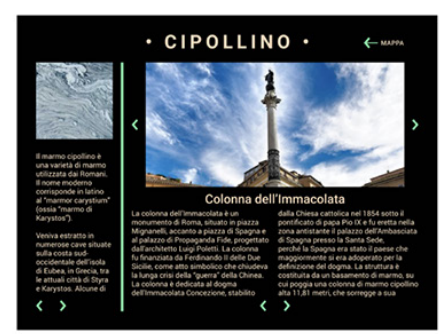
the different steps of Barillari, Paoli)

Fig. 5.Three-dimensional view of the interactive 


\section{Interactive Experience}

Interactive experiences, which expect a mutual exchange of input and feedback between user and machine, are divided into two categories: analog experiences that are based on an emission of an input by the user through direct contact with the machine (turn, press a button, lower or raise a lever, etc.); digital interactions that detect user movements through devices transforming an input into a machine action and not providing direct contact with the installation. Native gaming tools are used, often based on gesture recognition such as 3D leap motion, Microsoft Kinect, Nintendo Wii, etc. For an effective interactivity, in which response times and machine's speed of execution are fundamental, graphic representation must have a fair balance between the number of polygons of the modeled elements, display capacity and processing speed of inputs received.

Interactive installation I Marmi di Roma (Barillari, Paoli, DCVM I8-19) takes advantage of the analogue interactive mode to revolutionize the display model of marble samples in Geology section of MUST Sapienza, which currently is presented as a showcase in which the marbles are exposed together with an identification plate and an image of buildings / monuments in which that marble is used. Through an RFID recognition system applied on samples and a touch screen, users can choose the type of marble and explore its "geological" characteristics as well as navigate a map of Rome with possibility of deepening the marble ways of utilization in certain monuments or buildings.

Fig. 6. Storyboard illustrating the phases of the experience in Augmented Space. (students: Carosi, Mirenda).
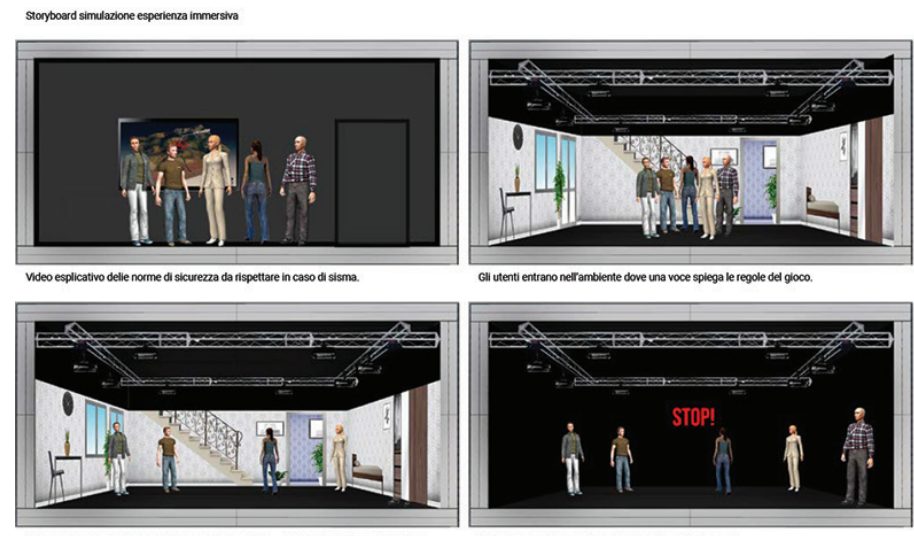

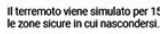
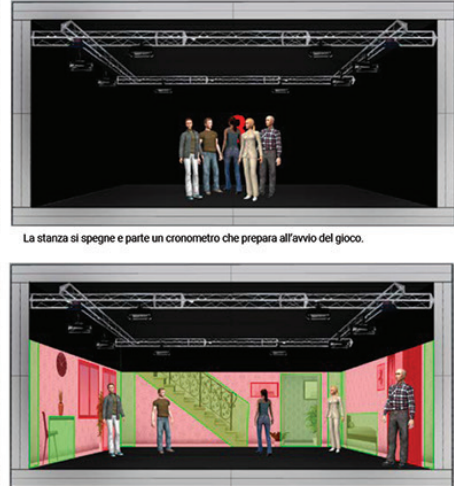

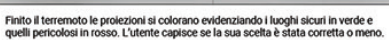

\section{Immersive Experience}

An immersive experience is a particular way of using content in which the observer is totally immersed in a space built or virtually reconstructed with which he can interact. The term immersion refers, from a perceptive point of view, to the ability of the aforementioned space to directly involve the senses of the subject, isolating it from the real environment stimuli. The immersive systems can be developed in Augmented Reality (AR), through the use of smart tools equipped with GPS and AR 'tracking' systems, allowing to superimpose real and virtual and letting users to act in it in real time by acquiring multiple types of information [Azuma 1997]. Or it can be developed in Virtual reality (VR) a system that instead simulates a space in which the user has the perception of finding himself inside a space, being able to move and to interact with the virtual objects. Augmented Space [Maniello 20 I8], is a third immersive modality, which originates from the overlap of information levels deriving from computer data within a real space. This translates into designing of immersive rooms in which, through projections, real space is enriched with content and information. 
Earthquake Room (Carosi, Mirenda, DCVM I8/19) is a project that aims to educate people both on the natural phenomenon of earthquake and on behavior to be adopted in the event of a seismic event. The experience is designed according to Augmented Space technology: users are located inside a room virtually furnished by projectors and, during the earthquake simulation time, they must be able to position themselves in correspondence of places considered safe, according to the safety rules on which they has been previously informed. At the end of the simulation, whose expected duration is 15 seconds, the projections on the wall will indicate to users what wrong positions (in red), and correct positions (in green) are.

Fig. 7. Images of the projections of two of the immersive room's walls: the upper part shows the state of the walls at the beginning of the game; the lower part illustrates, through the green and red coloring, the safe and dangerous spot that are activated when the game is over (students: Carosi, Mirenda).
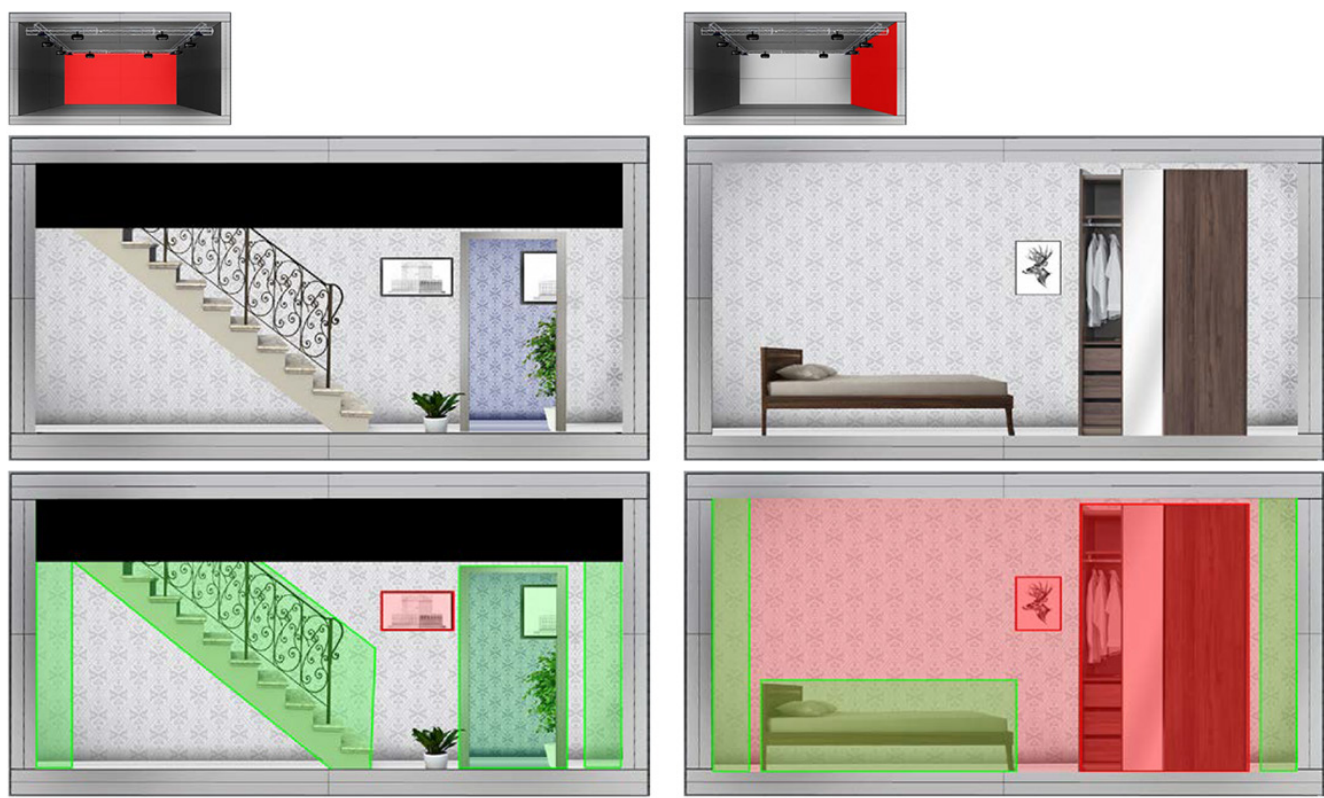

\section{Serious Game e Gamification}

Serious games come directly from videogames arena, but are developed for educational purposes. They are 'simulation' games (that faithfully reproduct some aspects of the real world) which, through solving puzzles or situations, stimulate learning and increase cultural accessibility by expanding the age of the user.

Gamification paths are very useful in exploring the museums. It is not a question of 'creating a game' but of experimenting with schemes and mechanisms linked to videogames to push users to perform certain actions, rewarding them intrinsically or extrinsically. Taking advantage of interactive learning, gamification represents an effective tool for conveying a message and inducing users to have active behaviors.

Interactive serious game Rock Seeker (Persiani, Milan, DCVM I 8/I9) recount from a particular point of view the Dattilioteca, a precious collection of rings belonging to Pope Leo XII made of valuable stones, exhibited at MUST Sapienza. The game allows the exploration of a virtual mine where users are invited to search for different stones that make up the papal rings. Once found, users can deepen the knowledge on the stones they'd discovered. Interaction takes place in an immersive room in which 3 walls analogically reproduce the game environment, while in the fourth the game is projected, playable with a classic controller. 


\section{Universal Design}

Universal Design can be described as a communication method that seeks to ensure the full usability of the exhibitions to all potential visitors by allowing a greater cultural accessibility [Empler 20 I8]. It is linked to the elimination of architectural barriers (both physical and sensorial), and has both a design and a representation component. The latter is linked to the sensory and cognitive disabilities and includes all those solutions for the visually impaired (who use touch as a means of communication and for which it have to structure a visual-tactile language) [Empler, Fusinetti 2019], hearing impaired (which make sight the privileged channel) [Empler 20I3] and cognitive impaired, that are used to simplify communication. These solutions must have a communication ability that makes even complex messages immediately recognizable and understandable.

The iSense Room is an installation conceived in an accessible way, an introductory part of the temporary exhibition of MUST Sapienza. A square-shaped room with a pedestal on each corner on which minerals are put and with which it is possible to interact: smelling (bituminous limestone), touching (quartz) and tasting (rock salt, which is supplied as sachets of kitchen salt). A central, protected, pedestal contains UVA rays sensitive minerals which are alternately illuminated to show how their color changes as changes the type of light. Installation is immersive, visitors find themselves in a dark room and are guided by a narrative voice that, aided by a double-wall video projection (designed with a visual translation for hearing impaired visitors), accompanies them in the exploration of planet Earth through the 5 senses. As the narration proceeds, the pedestals are gradually illuminated and each of them allows interaction.

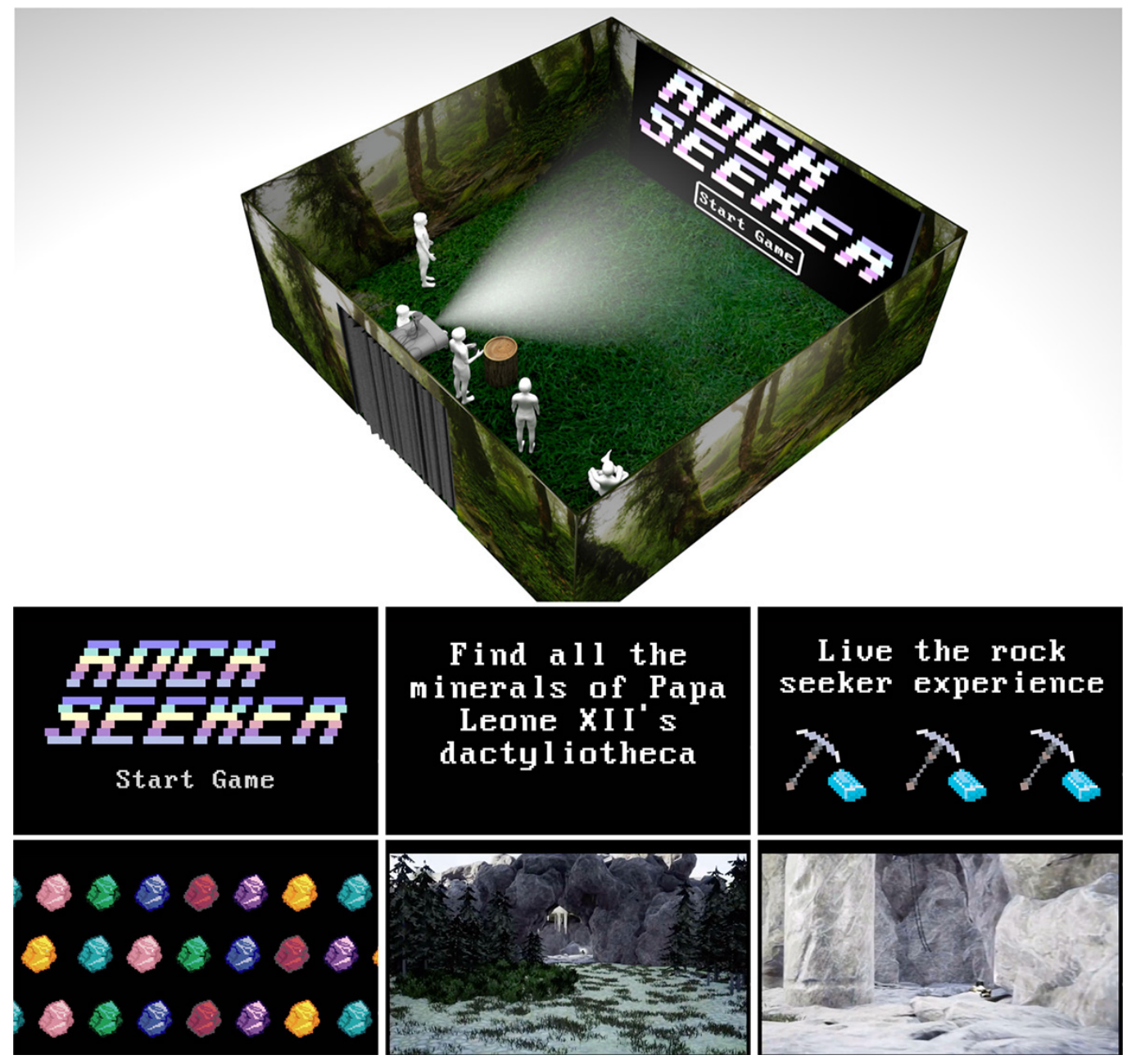


Fig. 9. Composition scheme of the iSense Room in which the interactions with the 5 senses are highlighted. (students: Neroni, Papi).
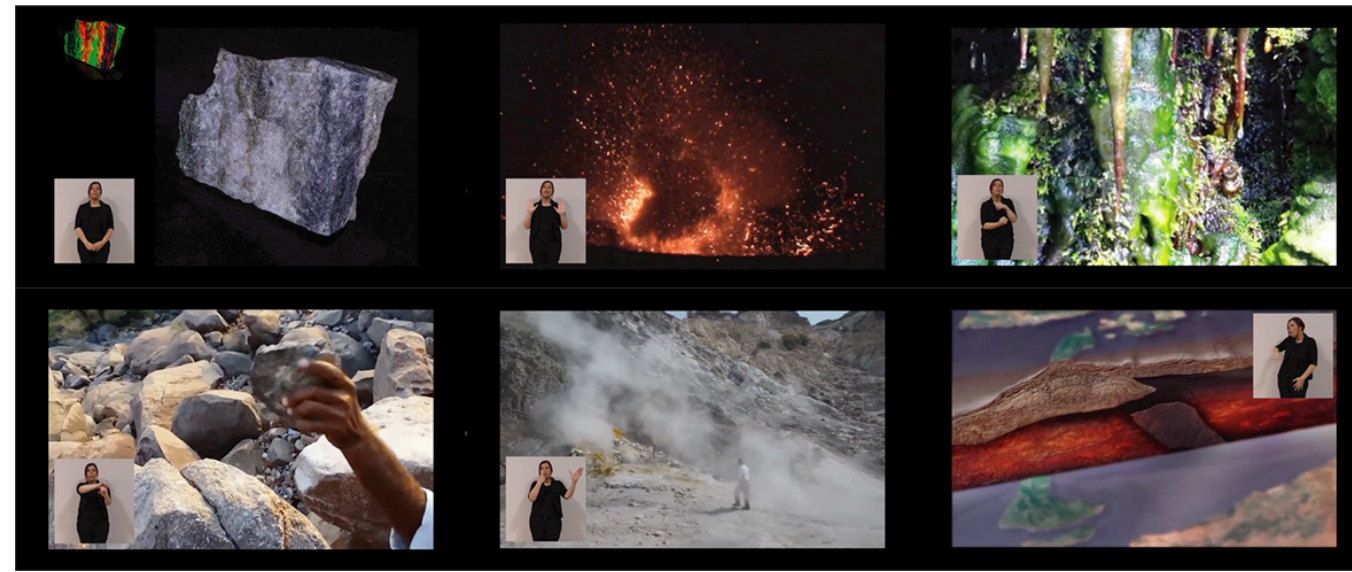

\section{Conclusion}

Transmedia museum designates a complex approach to communication and cultural access, denoting a growing attention from different disciplines (from representation, to sociology, to semiotics, to new media studies) that exploit a transversal perspective in order to involve a larger number of users. All the languages and tools described in the adopted methodology are not to be considered as separate sectors, but interconnected with different overlaps with the aim of increasing the offer of cultural assistance services through communication systems that effectively transmit the values of the heritage. If museum curators answer the question "what to communicate", information representation answer the questions "how to communicate' and 'to whom to communicate', taking on the role of connector between visitors' knowledge and learning process with specialist's acquaintance. These strategies, together with a strong focus on access, participation and representation, stimulate curiosity, interests and motivations during museum's exploration, activating participatory processes that allow to fully experience museum's experience.

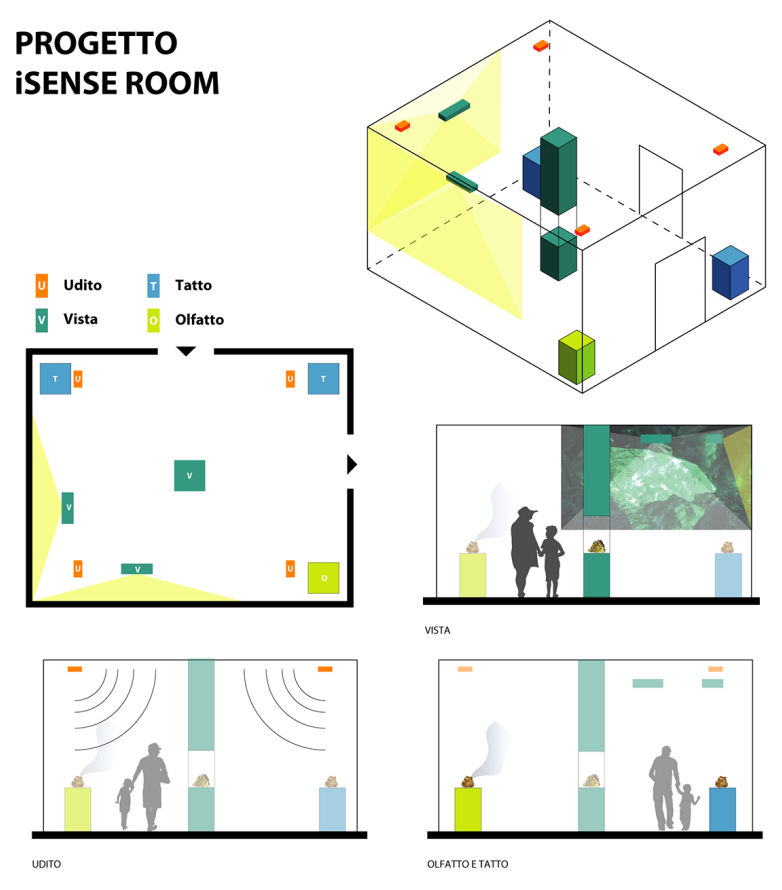

Fig. 10. Screenshot of the video with hearing impaired support (students:Neroni, Papi) 


\section{Notes}

[I] Master's Degree in Design Visual and Multimedia Communication, Computer Graphics and Multimedia Design I courses (course developed in collaboration with Lauren Ferro - Department of computer, automatic and management engineering Sapienza University of Rome).

\section{References}

Antinucci Francesco (20/4). Comunicare nel museo. Bari: Laterza.

Azuma Ronald T. ( 1997). A survey of augmented reality. In Presence:Teleoperators and Virtual Environment 6(4), pp. $355-385$.

Da Milano Cristina, Sciacchitano Erminia (20I5). Linee guida per la comunicazione nei musei: segnaletica interna, didascalie e pannelli. In Guido Manuel R. (a cura di). Quaderni della valorizzazione - NS I. Roma: Ministero dei Beni e delle Attività Culturali e del Turismo - Direzione Generale dei Musei - Servizio II - Gestione e valorizzazione dei musei e dei luoghi della cultura.

Empler Tommaso (20 I3). Universal Design: ruolo del Disegno e Rilievo. In Disegnare, Idee, Immagini, n. 46, pp......

Empler Tommaso (20I8). Traditional Museums, virtual Museums. Dissemination role of ICTs. In DisegnareCon. Advanced Technologies for Historical Cities Visualization, vol. I I, n. 2 I, p.......

Empler Tommaso, Fusinetti Alexandra (2019). Rappresentazione visuo-tattile. Comunicazione tattile per i disabili visivi. In Belardi Paolo (a cura di). Riflessioni, l'arte del disegno/il disegno dell'arte. Atti del $41^{\circ}$ Convegno Docenti delle discipline della rappresentazione, Perugia 19-2I settembre 2019. Roma: Gangemi Editore.

Jenkins Henry (20|4). Cultura convergente. Rimini: Maggioli editore.

Maniello Donato (20।8). Tecniche avanzate di video mapping. Spatial Augmented Reality applicata al bene culturale. Brienza: Le Penseur.

\section{Website}

Macvr3D.com, disposibile su: <https://macvr3d.com/>.

Mausoleodiaugusto.it, disponibile su: <http://www.mausoleodiaugusto.it/it/>.

The-uma.org, disponibile su: <https://the-uma.org/>.

Treccani, disponibile su: <http://www.treccani.it/enciclopedia/transmedia-storytelling_(Lessico-del-XXI-Secolo)/>.

\section{Authors}

Tommaso Empler, Sapienza Università di Roma, tommaso.empler@uniromal.IT

Adriana Caldarone, Sapienza Università di Roma, adriana.caldarone@uniromal.it

Alexandra Fusinetti, Sapienza Università di Roma, alexandra.fusinetti@uniromal.it

To cite this chapter. Empler Tommaso, Caldarone Adriana, Fusinetti Alexandra (2020). Musei tra narrazione, visualità e new media. Museums between narration, visuality and new media. In Arena A., Arena M., Brandolino R.G., Colistra D., Ginex G., Mediati D., Nucifora S., Raffa P. (a cura di). Connettere. Un disegno per annodare e tessere. Atti del $42^{\circ}$ Convegno Internazionale dei Docenti delle Discipline della Rappresentazionel Connecting. Drawing for weaving relationships. Proceedings of the 42th International Conference of Representation Disciplines Teachers. Milano: FrancoAngeli, pp. 3293-3312. 